\title{
Templates and Anchors for Antenna-Based Wall Following in Cockroaches and Robots
}

\author{
Jusuk Lee, Simon N. Sponberg, Owen Y. Loh, Andrew G. Lamperski, Robert J. Full, \\ and Noah J. Cowan, Member, IEEE
}

\begin{abstract}
The interplay between robotics and neuromechanics facilitates discoveries in both fields: nature provides roboticists with design ideas, while robotics research elucidates critical features that confer performance advantages to biological systems. Here, we explore a system particularly well suited to exploit the synergies between biology and robotics: high-speed antenna-based wall following of the American cockroach (Periplaneta americana). Our approach integrates mathematical and hardware modeling with behavioral and neurophysiological experiments. Specifically, we corroborate a prediction from a previously reported wallfollowing template - the simplest model that captures a behaviorthat a cockroach antenna-based controller requires the rate of approach to a wall in addition to distance, e.g., in the form of a proportional-derivative (PD) controller. Neurophysiological experiments reveal that important features of the wall-following controller emerge at the earliest stages of sensory processing, namely in the antennal nerve. Furthermore, we embed the template in a robotic platform outfitted with a bio-inspired antenna. Using this system, we successfully test specific PD gains (up to a scale) fitted to the cockroach behavioral data in a "real-world" setting, lending further credence to the surprisingly simple notion that a cockroach might implement a PD controller for wall following. Finally, we embed the template in a simulated lateral-leg-spring (LLS) model using the center of pressure as the control input. Importantly, the same PD gains fitted to cockroach behavior also stabilize wall following for the LLS model.
\end{abstract}

Index Terms-Bio-inspiration, biological system modeling, biomimicry, insect antenna, legged locomotion, wall following.

Manuscript received February 14, 2007; revised October 2, 2007. This paper was recommended for publication by Associate Editor P. Dario and Editor F. Park upon evaluation of the reviewers' comments. This work was supported by the National Science Foundation under Grant 0543985. The work of S. N. Sponberg was supported by a Fannie and John Hertz Foundation Graduate Fellowship. The work of O. Y. Loh was supported by a John Hopkins University Provost's Undergraduate Research Award. This paper was presented in part at the Society for Integrative and Comparative Biology (SICB), San Diego, CA, January 4-8, 2005, in part at the SICB, Orlando, FL, January 4-8, 2006, in part at the IEEE Conference on Robotics and Automation, Barcelona, Spain, April 18-22, 2005, in part at the First Ruperto Carola Symposium on Fast Motions in Biomechanics and Robotics: Optimization and Feedback Control, Heidelberg, Germany, September 7-9, 2005, and in part at the IEEE International Conference on Intelligent Robots and Systems, San Diego, CA, October 28-November 2, 2007.

J. Lee and N. J. Cowan are with the Mechanical Engineering Department, Johns Hopkins University, Baltimore, MD 21218 USA (e-mail: jsl@jhu.edu; ncowan@jhu.edu).

S. N. Sponberg and R. J. Full are with the Department of Integrative Biology, University of California, Berkeley, CA 94720 USA (e-mail: sponberg@ berkeley.edu; rjfull@berkeley.edu).

O. Y. Loh is with the Mechanical Engineering Department, Northwestern University, Evanston, IL 60208 USA (e-mail: o-loh@northwestern.edu).

A. G. Lamperski is with the Control and Dynamic Systems Program, California Institute of Technology, Pasadena, CA 91125 USA (e-mail: andyl@cds.caltech.edu).

Color versions of one or more of the figures in this paper are available online at http://ieeexplore.ieee.org.

Digital Object Identifier 10.1109/TRO.2007.913981

\section{INTRODUCTION}

$\mathbf{U}$ NDERSTANDING the control of locomotion presents challenges and opportunities to both biologists and engineers. On the biological side, we seek to identify general principles about how organisms extract salient sensory information, transduce this information into neural signals, and integrate them with the tuned dynamics of a locomoting musculoskeletal system to successfully navigate and maneuver in a complex environment. On the engineering side, we seek to design robust sensorimotor control strategies for robots that provide an environmental responsiveness to conditions outside of controlled laboratory settings. Here, we demonstrate how mutual, reciprocal inspiration can benefit both the fields of neuromechanics and robotics. In the context of a specific sensorimotor behavior - high-speed antenna-based wall following-we take a multileveled modeling and robotic development approach to elucidate the underlying biological phenomena. This, in turn, gives us novel strategies for the design and control of antennabased mobile robots.

Cockroaches demonstrate remarkable locomotor and navigational capabilities. An American cockroach (Periplaneta americana), running at up to $80 \mathrm{~cm} / \mathrm{s}$, can use its antennae as tactile probes to track a wall with no visual cues [1]-[3]. It can successfully navigate changes in wall orientation up to 25 times per second and can begin to respond to a wall angle change in as little as $30 \mathrm{~ms}$ [2]. Such extraordinary natural performance suggests that tactile sensors might provide a valuable feedback for robots operating with limited visual salience. More generally, a reliable encoding of sensory stimuli for steering maneuvers and integration of sensory signals with locomotor dynamics are basic requirements for autonomous robotic locomotion. As a biological investigation, antenna-based wall following lends itself to mathematical modeling at several levels as well as behavioral and neurophysiological experimentation.

A schematic overview of our research program, which includes modeling, robotic development, and experimental biology, is depicted in Fig. 1. Central to this program is a modeling hierarchy known as templates and anchors [4], [5]. A template is the simplest model that captures a desired behavior, while an anchor is a more complex, representative model of the behavior. Templates and anchors are not just "simple models" and "complex models"; there must be a natural embedding of the template behavior within the anchor [4]. For example, horizontal-plane locomotion in sprawl-postured animals such as cockroaches is well characterized by the lateral leg-spring (LLS) template [6]-[9]. Importantly, this template reveals that "internal" states (angular velocity and relative heading) can be 


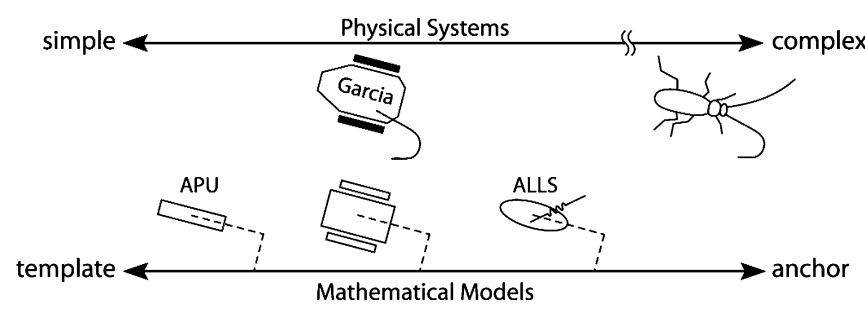

Fig. 1. Our research program involves multiple levels of modeling, robotic experimentation, ethology, and neurophysiology. Here, we present three models: the antenna-based planar unicycle (APU, Section II), a model for our Garcia robot (Section IV), and the antenna-based LLS (ALLS, Section V). Importantly, each level of modeling admits the same control structure, including parameters (up to scale), that was fitted to cockroach wall-following data [1]. The two physical systems involved in this study are an American cockroach (Section III) and a differential drive mobile robot (Section IV).

passively stabilized by mechanics alone, but "task-level" states (body position, angle, and forward speed) are neutrally stable and thus require active control [8], [10]. This paper addresses the antenna-based task-level control of body angle using three models along the template-anchor axis and the results from robotic and neurophysiological experiments.

We begin with a review of the simplest wall-following template (Section II), previously developed by three of the authors [1], which we build on through neurophysiology, robotics, and modeling (Sections III-V). A broader discussion (Section VI) integrates our specific results before a brief conclusion (Section VII).

\section{REVIEW: ANTENNA-BASEd PlANAR UNICYCLE (APU)}

The results of this paper are predicated on the multistride model by Cowan et al. [1] for high-speed wall following of $P$. americana. In their template model, antenna-based planar unicycle (APU), the antenna measures the lateral offset $d$ from a wall from some distance $\ell$ ahead of the running cockroach [Fig. 2(a) and (b)]; the lateral offset provides leeway for the swinging legs adjacent to the wall. The look-ahead distance $\ell$ gives the cockroach a preview of what is "ahead," affording time for the cockroach to avoid collisions with obstacles and protrusions from the wall. The offset $d$ is given by

$$
d=\ell \tan \theta+y \sec \theta
$$

where $\theta$ is the angle of the cockroach body relative to the wall and $y$ is the unicycle's center-of-mass (COM) distance from the wall. Their closed-loop model shown in Fig. 2(c) is given by

$$
\begin{aligned}
& \dot{y}=v^{*} \sin \theta \\
& \dot{\theta}=\omega \\
& \dot{\omega}=-\alpha \omega \underbrace{-K_{P}\left(d-d^{*}\right)-K_{D} \dot{d}}_{u}
\end{aligned}
$$

where $v^{*}$ is the forward running speed (constant), $\alpha=B / J, J$ is the animal's polar moment of inertia, $B$ is a damping coefficient, $K_{P}$ is a proportional gain, $K_{D}$ is a derivative gain, $d^{*}$ is a nominal "desired" wall-following distance, and $u$ is the inertia-scaled polar moment input that the cockroach must generate for turning [11]. The angular damping coefficient $B$ is a phenomenological parameter that captures the within-stride mechanical losses. (a)

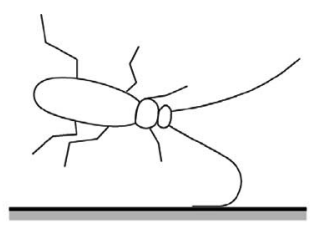

(b)

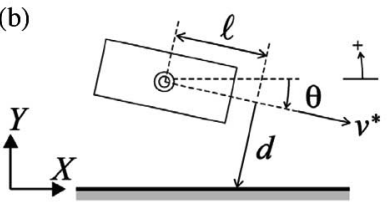

(c)

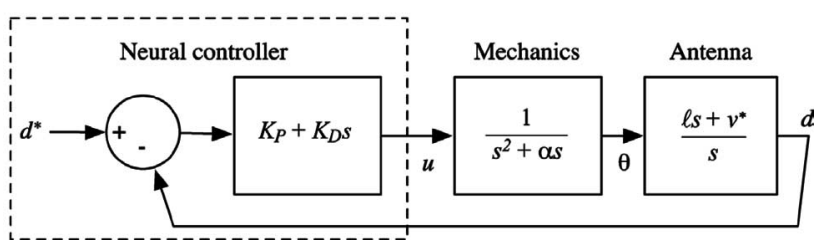

Fig. 2. (Adapted from [1]) (a) Depiction of a cockroach following a straight wall. (b) Unicycle model of the running cockroach. (c) Block diagram of simplified control model; $s$ is the complex frequency. The "mechanics" box represents the torsional dynamics. The "antenna" box is a simplified model of the antenna sensing kinematics. Cowan et al. [1] fit a simplified neural controller (in the dashed box) to their experimental data.

TABLE I

PARAMETER VAluES For P. AMERICANA AND the GARCIA RoBot

\begin{tabular}{c|ccccc}
\hline & $\begin{array}{c}v^{*} \\
(\mathrm{~m} / \mathrm{s})\end{array}$ & $\begin{array}{c}\ell \\
(\mathrm{m})\end{array}$ & $\begin{array}{c}\alpha \\
\left(\mathrm{s}^{-1}\right)\end{array}$ & $\begin{array}{c}K_{P} \\
\left(\mathrm{~m}^{-1} \mathrm{~s}^{-2}\right)\end{array}$ & $\begin{array}{c}K_{D} \\
\left(\mathrm{~m}^{-1} \mathrm{~s}^{-1}\right)\end{array}$ \\
\hline$P$. americana & 0.352 & 0.027 & 12.7 & 2600 & 433 \\
Garcia & 0.5 & $\mathbf{0 . 1 3 9}$ & 3.53 & $\mathbf{3 9 . 1 6}$ & $\mathbf{2 3 . 4 9}$ \\
\hline
\end{tabular}

Cowan et al. showed stable wall following of their model requires more than proportional $(\mathrm{P})$ control. To test this prediction, they obtained parameters $\left(\ell, \alpha, K_{P}, K_{D}\right)$ by fitting (2) to the behavioral data from cockroaches performing high-speed wall following and then tested the statistical significance of $K_{D}$. Their fitted parameter values for a specific speed group are given in Table I (first row). As predicted, P control was insufficient, and the next-simplest control law, proportional-derivative (PD) control, most parsimoniously accounted for their data.

\section{Cockroach Antenna NeURAL ReCORDINGS}

The analysis of the APU model with actual turning maneuvers of P. americana (Section II and [1]) supports a PD-control hypothesis, while rejecting P-only control. In this section, we directly examine if the cockroach's primary antennal receptors could encode both position and velocity information with appropriate temporal filtering for the wall-following behavior.

\section{A. Neurophysiology of the Cockroach Antenna}

Arthropod antennae are complex multimodal sensory structures containing mechanical, chemical (e.g., humidity and pheromone) and sometimes thermal receptors along the antennae [12], [13]. In cockroaches, antennal mechanosensory reception mediates many locomotor behaviors including wall following, escape, and slow-speed exploration [1], [2], [14]-[20]. The two most proximal antenna segments, the scape and pedicle, are actuated at their base joints and have a high density of mechanoreceptors for detecting the antenna base angle used mostly during exploration and escape [14], [20]. The remaining segments are called the flagellum-the long, 
slender, unactuated, and compliant portion of the antennawhich is the dominant source of tactile feedback information used for controlling the cockroach's body angle during wall following [2].

While robotic systems can filter the measurement $d$ [in Fig. 2(b)] to estimate $\dot{d}$, biological mechanoreceptors may directly encode both position and derivative terms. This encoding is often evidenced in a short-time constant, phasic response resolving to a longer time constant, relatively tonic response in neural recordings [15]. Camhi and Johnson [2] empirically demonstrated that receptors on the flagellum of the cockroach's antenna were both necessary and sufficient for successful wall following. The antennal nerve running along the central core of the antenna and into the brain carries all afferent (sensory) neurons from flagellar sensors. In addition to chemosensors, these sensors include three classes of mechanoreceptors distributed along the flagellum's length: campaniform sensilla, marginal sensilla, and mechanoreceptive hairs [21]. The specific set of mechanoreceptors that mediates wall following is unknown but activity in the antennal nerve can capture the responses of each of the possible candidates.

The rapid onset of the cockroach's turning response following antennal stimulus, as little as $30 \mathrm{~ms}$, suggests that limited processing time is available for computing controller inputs in the central nervous system (CNS). This constraint is made more severe since up to $20 \mathrm{~ms}$ of the processing time is required simply to conduct the signals down the proximal portion of the long flagellum, assuming typical conduction velocities of $\sim 1-4 \mathrm{~m} / \mathrm{s}$ for nongiant neurons (suggested in [22] and [23], and unpublished data). These considerations suggest that mechanoreceptor signals in the antennal nerve may directly encode the information available to the biological controller with appropriate temporal filtering, thus simplifying downstream processing.

We hypothesize that a cockroach's antennal nerve has a response typical of PD control. In the next subsection, we explicitly test if the neural response of antennal mechanoreceptors exhibit slow and fast time constant responses consistent with position and velocity encoding by recording the summed activity of the antennal nerve during wall deflection. If the transduction of mechanical stimuli by the primary afferent neurons in the antenna is sufficient for PD control, then the temporal profile of the neural activity should relate directly to the kinematic responses observed in wall-following cockroaches, so we compare the neural recordings to wall-following behavior [1]. Alternatively, both P- and D-control signals could be generated from processing in the cockroach's CNS downstream of the antennal nerve. Second, even if PD-like signals are present in antennal mechanoreceptors, filtering these signals to provide effective motor commands for the observed ethological response could require subsequent processing in the CNS.

\section{B. Experimental Methods}

We restrained live cockroaches ventral side up on an elevated transparent gel plate (Fig. 3). The proximal third of the flagellum was lightly restrained against the plate using insect pin staples. A silver chloride ground wire was affixed with EEG paste to the

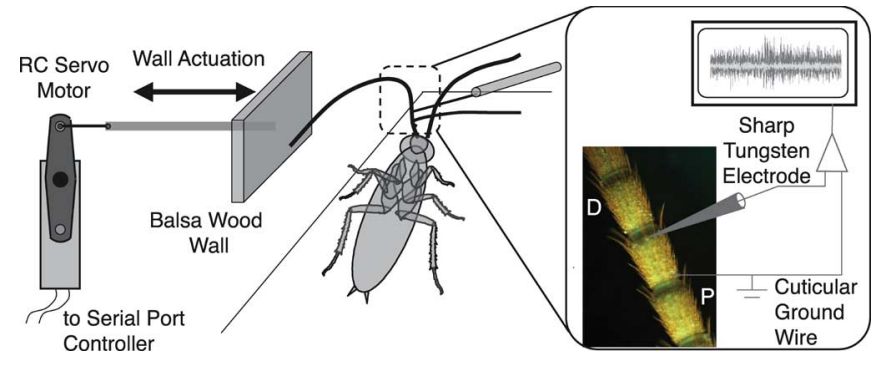

Fig. 3. Electroantennogram experimental setup: an $R C$ servo motor drives a wall segment against the free antenna of a restrained cockroach simulating a $30^{\circ}$ turn during fast locomotion $(\sim 45 \mathrm{~cm} / \mathrm{s})$. A tungsten electrode provides extracellular recordings from the antennal nerve (darker trace in inset), which are significantly above noise (lighter trace). The zoomed photo of the antenna is an autofluorescent image of three flagellar segments taken under a green fluorescent protein (GFP) filter to highlight the mechanosensory hairs, the largest on each segment. The distal (D) and proximal (P) labels indicate orientation.

antennal cuticle near the proximal restraint. A $5 \mathrm{M} \Omega$ tungsten extracellular electrode (A-M Systems, Carlsborg, WA) was inserted through the soft joint between two antennal segments so as to contact the antennal nerve distal to the ground electrode. The distal two thirds of the antenna was unconstrained and positioned in a bent "C" shape against an actuated wall segment of balsa wood. Overall, the orientation of the antenna mimicked positioning during wall following. The wall segment was driven by an $R C$ servo motor (JR Servo, Champaign, IL) via a two-pin linkage to perform a $7.7 \mathrm{~mm}$ linear displacement in $40 \mathrm{~ms}$. This resulted in movement equivalent to the "fast" wall-following trials reported in [1] (Fig. 4). In these trials, a cockroach executed a $30^{\circ}$ turning response while wall following at $46 \mathrm{~cm} / \mathrm{s}$. After the antenna had deflected for several seconds, we provided an equal displacement in the reverse direction. Altogether, we conducted 200 trials with four animals consisting of both an inward and outward push-and-hold of the antenna.

We recorded a multiunit response from the antennal nerve since the specific mechanoreceptor(s) mediating wall following was unknown; this recording method provided access to all mechanoreceptive signals in the antennal nerve. Multiunit, extracellular recordings of neurons tend to underestimate the power of the neural signal due to destructive interference of out-of-phase spiking events. These recordings are, therefore, likely to be conservative indicators of changes in neural activity.

Although chemosensory responses may be present as background activities, the mechanoreceptive responses observed here were not due to contact chemoreception as we observed similar responses to wall deflection when we used an insect pin to manually deflect the antenna. We also controlled for windinduced sensor activity as well as electrical and mechanical coupling between the motor and the recording apparatus by driving the wall under normal experimental conditions, but without a direct contact with the antenna. In these cases, we observed no change in neural activity. No musculature exists in the antenna flagellum and so efferent (motor) neurons are absent in antennal nerve recordings taken from the flagellum.

We computed overall activity in the antennal nerve by computing the rms power of the signal using small overlapping 


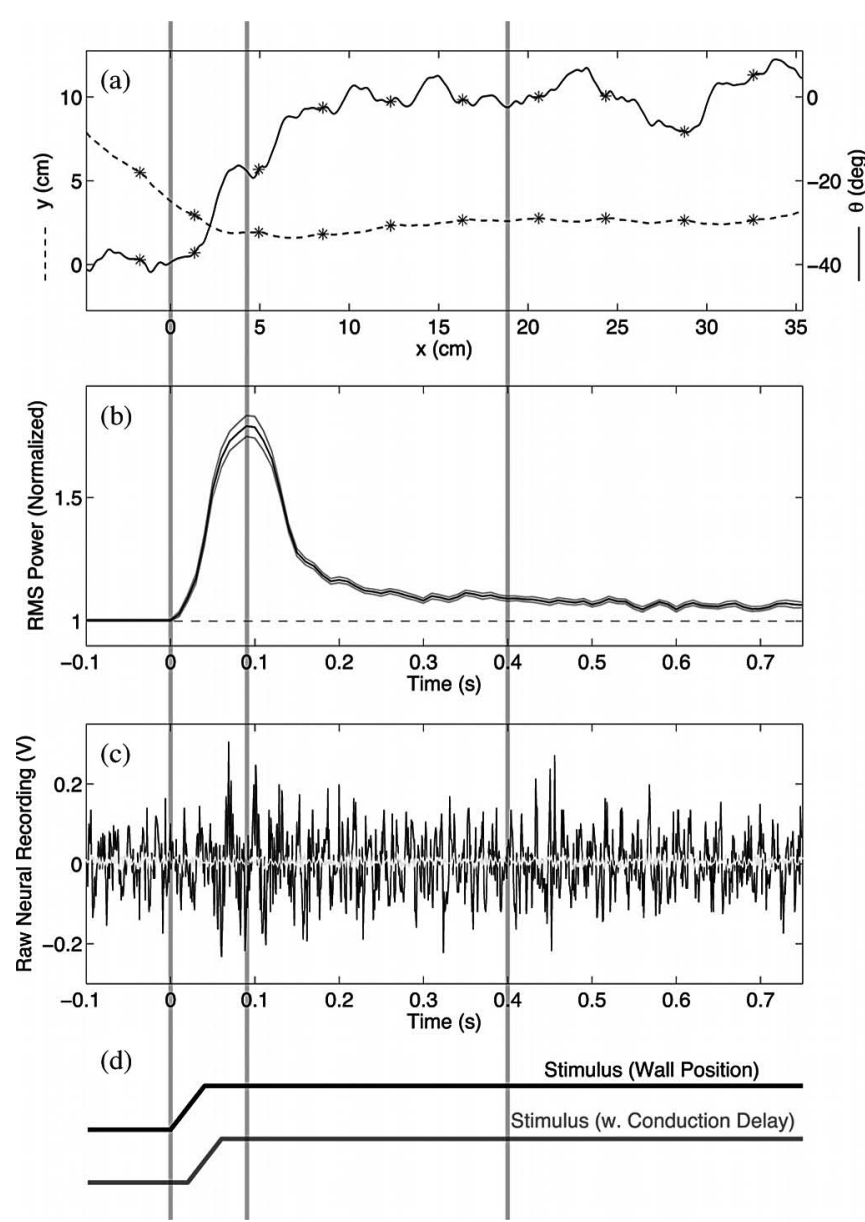

Fig. 4. Comparison of the antenna neural recording to the kinematic data of a running cockroach. (a) Position (dashed) and orientation (solid) of a cockroach running along a wall [1]; strides are marked by asterisks $(*)$. (b) Normalized neural rms power [mean (black) with confidence interval (gray)] of the antenna when the wall approached toward the cockroach and stopped; it has been scaled to approximately match the kinematic traces above. (c) Raw neural recording voltage (black) and background voltage (gray). (d) Time trace of the actual wall displacement (black) and delay-accounted wall displacement (gray). The vertical lines indicate (from left to right) the stimulation onset, rms peak time, and settling time.

windows of $20 \mathrm{~ms}$ at $10 \mathrm{~ms}$ intervals. Individual action potentials were not differentiated in the bulk recording although future investigations of the specific mechanoreceptors mediating this response will likely require such recordings. All trials were normalized relative to the activity measured with the artificial wall stationary in its zero position.

In the behavioral experiments, as the cockroach returns to its preferred distance from the wall, we would expect the P signal (tonic firing rate) to return to its baseline value. In the electrophysiological experiments, by contrast, the antenna was intentionally held in a deflected position to observe any changes in the plateau neural activity that would be consistent with position encoding. These data [Fig. 4(b)], therefore, do not directly mimic the activity seen during closed-loop wall following [Fig. 4(a)].

\section{Neural Recording Results}

The rms response had two phases: an initial short timeconstant phasic response and then resolving to a more tonic response (Fig. 4). The initial phasic response showed a large increase in neural activity (paired $t$-test, $p$-value $<0.001$ ) peaking at $\sim 180 \%$ of baseline power around $100 \mathrm{~ms}$ after the onset of stimulation. Neural activity then decreased until reaching a long time constant, effectively "steady-state" response 300-500 ms after stimulation. The steady state was dependent on the displacement direction, showing a significant increase $(1.033 \pm 0.004)$ in firing compared to the original baseline $(1.000 \pm 0.002)$ if the antenna was deflected toward the cockroach and a significant decrease $(0.977 \pm 0.003)$ compared to the baseline $(1.001 \pm 0.005)$ if the wall moved away from the cockroach (both paired $t$-tests, $p$-values $<0.001$ ). This combined phaso-tonic response to wall deflections of the antenna is consistent with encoding of both velocity and position information, respectively.

In all cases, the neural activity near the base of the flagellum was significantly above baseline $20 \mathrm{~ms}$ after the onset of deflection. This delay almost certainly results from the conduction velocity (about $1 \mathrm{~m} / \mathrm{s}$ ) from the region of deflection to the recording site (about $2 \mathrm{~cm}$ ).

The time course of the neural response closely matched the turning kinematics observed in the wall-following cockroaches (Fig. 4). In the kinematic trials, active turning began about $40 \mathrm{~ms}$ after initial deflection, consistent with a fast, but reasonable, processing delay from sensory reception in the brain to changes in leg ground reaction forces. The phasic neural response, putative derivative control signal, peaked at the time when the cockroach would be producing significant moments around the COM to generate a turn ( $~ 90 \mathrm{~ms})$. The new steadystate firing rate, putative positional control signal, culminated approximately at the same time that the cockroach completed its correction in the kinematic trials $(\sim 300 \mathrm{~ms})$.

Our results support the hypothesis that the sensory systems in the flagellum encode both position and velocity information, and the time course of the neural response at this early stage is consistent with the kinematics of wall following.

\section{ROBOTIC INTEGRATION OF BIO-INSPIRED ANTENNA}

We found evidence for a PD-like control signal directly in the antennal nerve, but it is unclear whether the PD-controller gains fitted for the idealized APU model [1] accurately reflect those of the actual cockroaches.

We integrate a custom artificial antenna with a mobile robot platform to test the efficacy of using the APU as a template model for our biological system. Since the previous section suggests that sensory signals $d$ and $\dot{d}$ may indeed be available for cockroaches, we test whether the same PD-controller gains (up to a scale) that were fitted in [1] are sufficient for stable wall following in our robotic platform despite effects such as antenna-to-wall friction and nontrivial forward-speed dynamics. A positive result would indicate the sufficiency of the PD controller-including the specific gains fitted to the cockroach-despite the APU model neglecting many complexities inherent to real-world antenna-based wall following. 


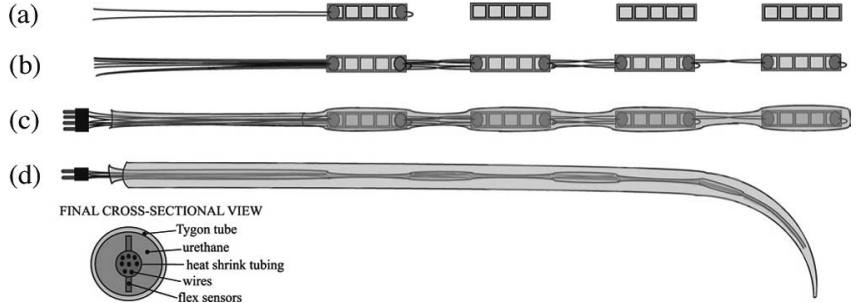

Fig. 5. Overview of the cast urethane antenna construction process. (a) Conductive epoxy attaches wire leads to both ends of each flex $2.5 \mathrm{~cm}$ sensor. (b) Two wires from each gauge run down the center to the base as close as possible to the neutral bending axis. (c) Heat-shrink tubing holds the gauges and wires in place leaving room for the wires to move freely. The wires at the base terminate at a single header that plugs into custom amplifiers on the robot. (d) Assembly slides into a preshaped (by heating until soft, forming, then cooling) Tygon rubber tube. A vacuum pump draws two-part urethane casting compound into the Tygon tube. The compound cures for $48 \mathrm{~h}$ prior to calibration. Teflon tape wrapped along the antenna reduces friction between the antenna and the wall.

\section{A. Antenna Design}

To provide tactile feedback to our mobile robot, we designed and built an artificial antenna (Fig. 5). The design is based on observations of cockroaches and their antennae while wall following [2], [12], [13], [24]. These observations and their implications toward our design are briefly summarized as follows.

1) $P$. americana may use bend (campaniform and marginal sensilla) and/or touch sensors (mechanoreceptive hairs) along the flagellum [12] to measure its pose relative to a wall. We integrated a series of flex sensors (cut from Abrams Gentile 4" FlexSensors) along the length of the antenna to obtain local curvatures of the antenna (Fig. 5).

2) Arthropod flagella decrease in stiffness along their length [13] allowing them to conform to surface irregularities. We tapered our antenna to achieve a similar stiffness profile. A rigid support in the middle of the antenna (see Fig. 6) restricted the second flex sensor from the base from bending.

3) We curved the tip of the antenna [Fig. 5(d)] to prevent the unidirectional flex sensors near the tip from bending forward. This also helped the antenna to conform smoothly to irregularities and protrusions of varying size.

4) We rigidly clamped the base of our antenna to the robot at about $35^{\circ}$ from the heading, similar to cockroaches during rapid wall following [see Fig. 12(d)].

The raw data provided by the antenna consist of an amplified voltage output $V_{i}$ from each flex sensor. To extract the distance to the wall $d$ from the voltage outputs, we performed a least-squares fit. ${ }^{1}$ Here, we do not consider the two proximal flex sensor measurements; the high stiffness near the base of the antenna and the addition of the support structure in the middle of the antenna have limited their motions and did not contribute significantly to the calculation of $d$. The omission of the most proximal sensory data is consistent with Camhi and Johnson's [2] result that wall

\footnotetext{
${ }^{1}$ While maintaining antenna-wall contact, we recorded voltages from each flex sensor as we varied the distance $d$ and the angle of the robot relative to the wall. We obtained a least-squares fit for the affine relationship $d=a^{T} V+b$. Subsequent work will include an elastica model of bending to calibrate contact distance as used for whiskers [25], [26]
}

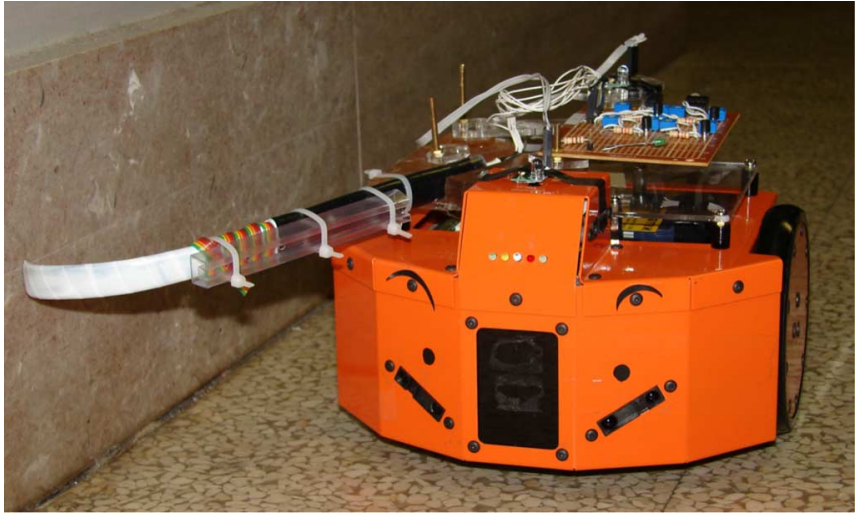

Fig. 6. Cast urethane antenna with embedded flex sensors mounted on the Garcia robot.

following requires distal receptors in the flagellum without the sensory data from the base.

\section{B. Integrating the Antenna With the Garcia Robot}

Our physical instantiation of the unicycle-like robot is called Garcia (Acroname, Inc., Boulder, CO) shown in Fig. 6. It is a three-wheeled robot, with two drive wheels sharing the same axis of rotation and a third passive omnidirectional wheel, with zero caster sweep space, for balance. This machine was used previously by Lamperski et al. [27] to demonstrate the feasibility of wall following using a multirigid-linked antenna as its distance sensor. An onboard XScale ARM processor updates our control law at $50 \mathrm{~Hz}$ using sensory information from the antenna. During each trial, we log internal states such as the voltages from the four flex sensors (and hence, the perceived distance to the wall) and the encoder-measured velocities of the two wheels. In addition, we use an overhead camera to obtain the ground-truth position of the robot for post analysis.

The primary difference between the theoretical unicycle model and the Garcia robot is the need for forward velocity control. One can show [27] that the dynamical equations for the robot are of the form

$$
\left[\begin{array}{c}
\dot{v} \\
\dot{\omega}
\end{array}\right]=A\left[\begin{array}{l}
v \\
\omega
\end{array}\right]+\overbrace{B\left[\begin{array}{l}
V_{1} \\
V_{2}
\end{array}\right]}^{u}
$$

where

$$
A=\left[\begin{array}{rr}
-\gamma & 0 \\
0 & -\alpha
\end{array}\right], \quad B=\left[\begin{array}{ll}
b_{1} & b_{1} \\
-b_{2} & b_{2}
\end{array}\right]
$$

Here, $V_{1}$ and $V_{2}$ are the input voltages for the left and right wheels, respectively, $v$ is the forward speed, and $\omega$ is the angular velocity of the robot. The parameters $\alpha, \gamma, b_{1}, b_{2}>0$ are expressed in terms of the armature resistance, the torque constant, the back electromotive force constant, a frictional damping constant for each wheel, the wheel radius, the lateral offset of each wheel from the center, the robot's moment of inertia in the yaw direction, the moment of inertia of each wheel, and the mass of the robot. We used the values $A$ and $B$ that Lamperski et al. [27] fitted for the robot. 
For wall following, we combined the antenna-based PD controller suggested by the APU model (Section II), together with a proportional-integral forward-speed controller

$$
\left[\begin{array}{l}
u_{1} \\
u_{2}
\end{array}\right]=\left[\begin{array}{l}
-K_{V}\left(v-v^{*}\right)-K_{I} \Sigma \\
-K_{P}\left(d-d^{*}\right)-K_{D} \dot{d}
\end{array}\right], \quad \Sigma=\int_{0}^{t}\left(v-v^{*}\right) d t
$$

where $v^{*}$ is the desired forward speed and $d^{*}$ is the desired distance to the wall. To map the torques into motor voltages, the control input (4) is written as $V=B^{-1} u$, since $B$ is invertible.

Inserting (4) into (3), we have

$$
\left[\begin{array}{c}
\dot{y} \\
\dot{\theta} \\
\dot{\Sigma} \\
\dot{v} \\
\dot{\omega}
\end{array}\right]=\left[\begin{array}{c}
v \sin \theta \\
\omega \\
v-v^{*} \\
-\gamma v-K_{V}\left(v-v^{*}\right)-K_{I} \Sigma \\
-\alpha \omega-K_{P}\left(d-d^{*}\right)-K_{D} \dot{d}
\end{array}\right]
$$

where $d$ is the same as in (1). Linearizing the system at its equilibrium point $(y, \theta, \Sigma, v, \omega)=\left(d^{*}, 0,-\alpha v^{*} / K_{I}, v^{*}, 0\right)^{T}$, we obtain the characteristic polynomial

$$
\begin{aligned}
p(s)= & \left(s^{2}+\left(\gamma+K_{V}\right) s+K_{I}\right)\left(s^{3}+\left(\alpha+K_{D} \ell\right) s^{2}\right. \\
& \left.+\left(K_{P} \ell+K_{D} v^{*}\right) s+K_{P} v^{*}\right) .
\end{aligned}
$$

The second-order polynomial factor has negative roots if

$$
K_{V}>-\gamma \text { and } K_{I}>0
$$

and, by Routh's stability criterion, the latter third-order polynomial factor has negative roots if

$$
K_{P}>0, \quad K_{D}>-\frac{\alpha}{\ell}, \quad \text { and } \quad \frac{K_{D}}{K_{P}} v^{*}>\frac{v^{*}}{\alpha+K_{D} \ell}-\ell
$$

where $\gamma, \alpha, v^{*}, \ell>0$. Notice that the conditions for forward stability-which are constraints on $K_{V}$ and $K_{I}$ - are decoupled from the conditions on rotational stability - which are constraints on $K_{P}$ and $K_{D}$.

\section{Dynamically Scaled Parameters of P. americana}

We found the necessary parameters for the Garcia robot using the principle of similitude; the APU's torsional dynamics equation (2) and the last row of (5) are identical, namely they are of the form

$$
\dot{\omega}=-\alpha \omega-K_{P}\left(d-d^{*}\right)-K_{D} \dot{d}
$$

where $\alpha, K_{P}$, and $K_{D}$ are known quantities for the cockroach [1]. Selecting two fundamental quantities, $v$ and $\ell$, leads to the following dimensionless ratios:

$$
\widetilde{\alpha}=\frac{\alpha \ell}{v^{*}}, \quad \widetilde{K}_{P}=\frac{K_{P} \ell^{3}}{v^{* 2}}, \quad \widetilde{K}_{D}=\frac{K_{D} \ell^{2}}{v^{*}} .
$$

Setting the desired velocity for the Garcia robot to be $0.5 \mathrm{~m} / \mathrm{s}$ and assuming $\alpha$ to be constant, we calculated the dimensionally scaled look-ahead distance for the Garcia robot. We found values for $K_{P}$ and $K_{D}$ in a similar way. The calculated values are shown in bold in Table I (second row). (a) Fwd vel, v (m/s)

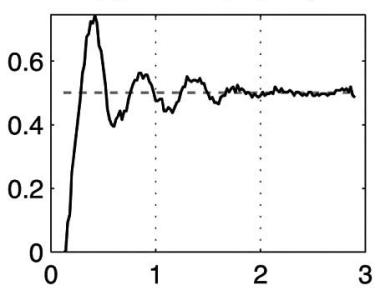

(c) Body angle (deg)

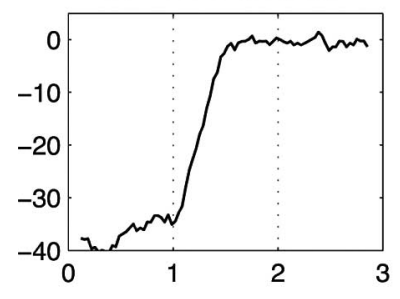

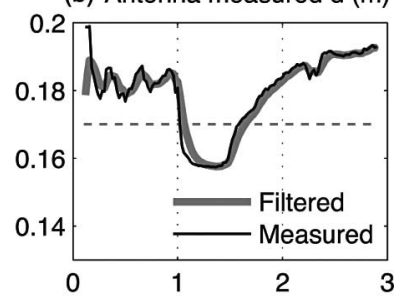

(d) Body position, y (m)

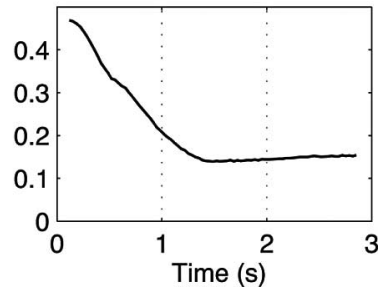

(b) Antenna measured d (m)

Fig. 7. Garcia robot's internal states during a trial with parameters from Table I. The onset of the angled wall contact occurs at $t=1 \mathrm{~s}$. (a) Measured forward speed (solid) with $v^{*}=0.5 \mathrm{~m} / \mathrm{s}$ (dashed). (b) Measured raw (solid black) and filtered (solid gray) $d$ values with $d^{*}=0.17 \mathrm{~m}$ (dashed); to reduce noise, we low-pass filtered raw distance measurements: $d_{k}=\lambda d_{k-1}+(1-\lambda) d_{\text {raw }, k}$, where $\lambda=0.7$ (trial and error); $\dot{d}$ was estimated via finite difference. (c) and (d) Body angle and position obtained from overhead camera images.

\section{Experimental Results of Robotic Wall Following}

The experimental setup for the Garcia robot was similar to that of the cockroach behavioral experiment by Cowan et al. [1]. The robot followed a wall that consisted of a straight control wall to allow the robot to reach its steady state and an angled wall to act as a "step" perturbation to the internal state $\theta$. We used the parameter values for the Garcia robot shown in Table I with $K_{V}=1 \mathrm{~s}^{-1}$ and $K_{I}=1 \mathrm{~s}^{-2}$. This set of parameters satisfied stability conditions (7) and (8).

We ran 30 trials at $v^{*}=50 \mathrm{~cm} / \mathrm{s}$ with the $30^{\circ}$ angled wall demonstrated successful turning. Figs. 7 and 8 show a subset of states that were collected during a typical trial; the rest of the trials were very similar. Our somewhat arbitrary choice of speed gains $\left(K_{V}, K_{I}\right)$ produced substantial oscillations in the forward speed, but, nevertheless, reached steady-state speed within about $2 \mathrm{~s}$ and did not imperil wall-following performance. The body-angle transient responses for the APU model and the Garcia robot were comparable (Fig. 12): the rise time, peak time, and overshoot for the Garcia was $0.60 \mathrm{~s}, 1.23 \mathrm{~s}$, and $2.5 \%$, respectively. For the APU from [1], dimensionally mapped into Garcia's scale, these were $0.50 \mathrm{~s}, 1.20 \mathrm{~s}$, and $17.3 \%$.

The Garcia robot failed to negotiate turns of angles greater than about $40^{\circ}$ because the distal end of the rigid antenna support catches the angled wall, forcing the robot to turn inward toward the wall. We believe that this problem will be addressed through the design of more flexible antennae with more appropriately tapered mechanical stiffness.

Typically, the robot followed the wall with a constant error in $d$ : in Fig. 7(b), the robot maintains the measured distance of $0.18-0.19$ (solid) despite the commanded value $d^{*}$ of 0.17 (dashed). This was likely caused by nonnegligible forces produced by the artificial antenna against the 
(a) Flex sensor 1 (base)

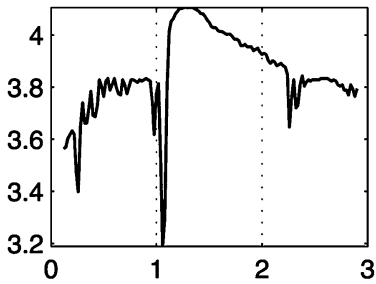

(c) Flex sensor 3

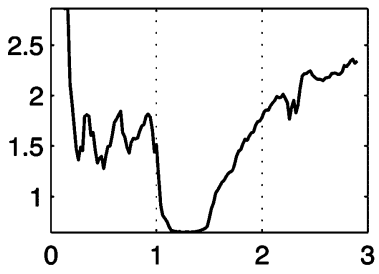

(b) Flex sensor 2

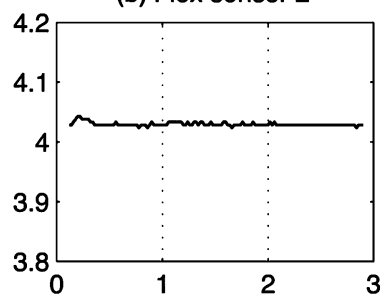

(d) Flex sensor 4 (tip)

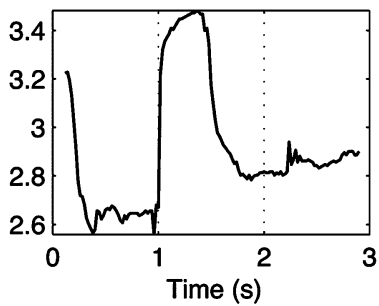

Fig. 8. Raw amplified voltage values from the four flex sensors embedded in our antenna; they are shown for their qualitative form. The flex sensor at the base of the antenna (a), which is not used to calculate the value $d_{\text {raw }}$ in Fig. 7(b), shows the onset of the angled wall contact with a sharp peak. The second flex sensor from the base (b) registers zero motion because it is anchored to the rigid support base discussed in the main text. The two distal flex sensors (c) and (d) are used to calculate the measurement $d_{\text {raw }}$ shown in Fig. 7(b). The third flex sensor (c) flexes more (indicated by the decrease in voltage values) as it encounters the initial part of the angled wall. Meanwhile, the remaining distal part of the antenna (d) conforms to the wall by decreasing its flexion (indicated by the increase in voltage values).

wall ${ }^{2}$ : adding a torsional spring term $c\left(d-d_{\max }\right)$ to the last line of (5) shifts the equilibrium distance from the wall to $\left(K_{P} d^{*}+c d_{\max }\right) /\left(K_{P}+c\right)$. For negligible stiffness $c$, the equilibrium distance is $d^{*}$ as expected.

Fig. 8(c) and (d) shows that flex sensors 3 and 4 do not necessarily return to their original configuration after encountering the angled wall. One possible explanation could be that there is a range of "stable" configurations of the antenna for a given $d$ due to friction, memory effects, or other factors. Reasonable calibration can cope with this, as suggested by the fact that the Garcia, despite the drift, successfully followed the wall after making the turn.

\section{ANTENNA-BASEd LATERAL LEg SPRING}

In the previous models, the control input is literally the torque applied to the body through a continuous actuation. A fundamental question that remains is how such a control input might be applied to control stride-to-stride dynamics in a legged organism. To guide further development in maneuverable legged robots and generate hypotheses for how the biological system modifies its motor output, we require a more representative, anchored mathematical model than our APU model. As a first step toward answering these questions, we add antenna-based control to a horizontal-plane legged model of cockroach running.

\footnotetext{
${ }^{2}$ To quantify cockroach antenna reaction forces, we used the force levers that Dudek and Full [28] used to measure passive leg forces in cockroaches; the antennal forces fell below the noise floor of the sensors. This (and our casual observation) suggests that cockroach antennal forces are negligible compared to leg forces.
}

(a)

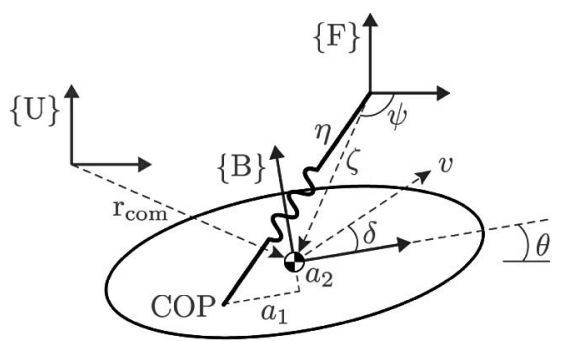

(b)

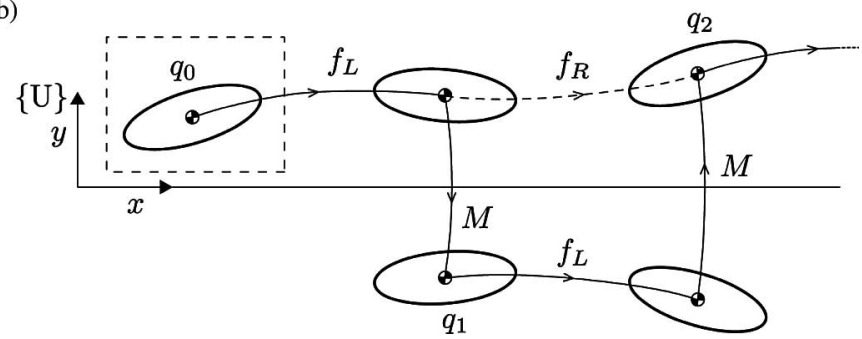

Fig. 9. (a) Schematic model of the LLS model; $\theta$ is the body angle w.r.t. the $x$-axis in inertial frame $\{\mathrm{U}\}, v$ is the speed of the COM, $\delta$ is the velocity angle w.r.t. the $x$-axis of the body frame $\{\mathrm{B}\}, \zeta$ is the distance from the foot placement to the COM, $\psi$ is the angle from the $x$-axis of the foot frame $\{\mathrm{F}\}$ to the COM, $\eta$ is the leg length, $\left[a_{1}, a_{2}\right]^{T}$ is the location of the COP written in $\{B\}$. (b) Illustration of multistep dynamics and its equivalent representations. (The spring legs are not depicted for presentation purposes.)

\section{A. LLS Model}

Schmitt and Holmes [29], [30] introduced the horizontal plane, lateral leg-spring (LLS) model to study running sprawlpostured animals that exhibit lateral oscillations during each stride. The model is a rigid body on a frictionless surface that has two massless, spring-loaded, telescoping legs, as shown in Fig. 9(a). The legs attach to the body at a point called the center of pressure (COP) and can rotate freely about that point. Cockroaches run in a tripod gait; hence, each virtual leg represents the combined effect of three stance legs.

At the start of a left step, the left virtual leg affixes its foot to the ground with leg base angle $\beta_{0}$ and with relaxed leg length $l_{0}$. Due to the body's initial velocity, the leg gets compressed while rotating about its foot and the COP. The compressed leg generates a counter force to the body along the leg and through the COP. As the body moves ahead of the foot contact point, the spring starts to decompress and pushes the body forward. When the left leg returns back to its relaxed length $l_{0}$, the left leg lifts off while simultaneously the right leg touches down (at an angle $-\beta_{0}$ ), and the right step ensues.

Since the LLS model is energy conserving and SE(2) (special Euclidean group) invariant, the model can, at most, exhibit partial asymptotic stability only in the COM direction with respect to the relative heading $\delta$ and angular velocity $\omega \equiv \dot{\theta}$. The COM speed $v$ and body pose in $\mathrm{SE}(2)$ are neutrally stable.

The position of COP is critical to the LLS's stability. Suppose the COP position for a left-step is parameterized by

$$
\left[\begin{array}{l}
a_{1} \\
a_{2}
\end{array}\right]=\left[\begin{array}{l}
b_{1}+c_{1}(\psi-\theta) \\
b_{2}+c_{2}(\psi-\theta)
\end{array}\right]
$$

where $a_{1}$ and $a_{2}$ are along $x$ - and $y$-axis of the LLS body frame $\{\mathrm{B}\}$ and $b_{i}$ 's and $c_{i}$ 's are constants. A special case of 


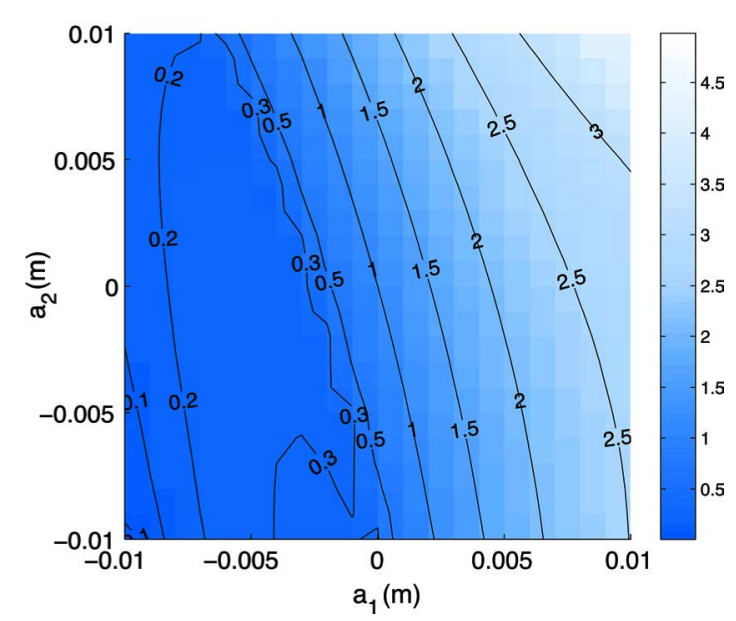

Fig. 10. Maximum nonunity eigenvalue for the linearized return map at various fixed-COP positions around the COM. Parameters used for P. americana are shown in the caption of Fig. 11.

this parameterization is when $a_{2}=0$ and $c_{1}=0$ [29], [30]: if $a_{1}<0, \delta$ and $\omega$ are asymptotically stable; if $a_{1}=0$, $\omega$ becomes neutrally stable; if $a_{1}>0$, the system becomes unstable. When $a_{1}<0$, the body mechanics alone can stabilize the LLS system [29]. However, a fixed COP on the fore-aft axis yields yaw dynamics that do not match biological data. Placing the COP laterally offset to the side $\left(a_{2}=\right.$ constant $\left.<0\right)$ better matches the yaw dynamics, and the system can achieve stability even if the COP lies in front of the COM as long as it is appropriately offset to the side [31], as shown in Fig. 10. Henceforth, we restrict ourselves to $c_{1}=c_{2}=0$, in which case the Hamiltonian for a left step can be written as

$$
H=\frac{p_{\zeta}^{2}}{2 m}+\frac{p_{\psi}^{2}}{2 m \zeta^{2}}+\frac{p_{\theta}^{2}}{2 I}+\frac{k\left(\eta-l_{0}\right)^{2}}{2}
$$

from which the equations of motion are

$$
\begin{aligned}
& \dot{\zeta}=\frac{p_{\zeta}}{m}, \quad \dot{p}_{\zeta}=\frac{p_{\psi}^{2}}{m \zeta^{3}}-\frac{k\left(\eta-l_{0}\right)}{\eta}\left(\zeta-a_{2} \cos \phi+a_{1} \sin \phi\right) \\
& \dot{\psi}=\frac{p_{\psi}}{m \zeta^{2}}, \quad \dot{p}_{\psi}=-\frac{k\left(\eta-l_{0}\right)}{\eta}\left(\zeta a_{1} \cos \phi+\zeta a_{2} \sin \phi\right) \\
& \dot{\theta}=\frac{p_{\theta}}{I}, \quad \dot{p}_{\theta}=-\dot{p}_{\psi}
\end{aligned}
$$

where $\phi=\psi-\theta$ and $p$ 's are the conjugate momenta. The leg length $\eta=\eta\left(\zeta, \psi, \theta, a_{1}, a_{2}\right)$ can be determined from Fig. 9(a).

\section{B. Hybrid Step-to-Step Dynamics}

For task-level control of the multistride dynamics, we seek a compact representation of the step-to-step dynamics. Let $\left\{A_{k}\right\}$ denote the location of the body frame at the beginning of the $k$ th step. In other words, $g_{k}$ is the transformation from $\left\{A_{k}\right\}$ to the world frame $\{U\}$. For $k$ odd, we take a mirror image around the $x$-axis of frame $\left\{A_{k}\right\}$ at the beginning of the $k$ th step ( $k$ odd), simulate the dynamics using the equations of motion for a left step and then take another mirror image around the $x$-axis. In this way, the right step map is computed in terms of the left one. This can be expressed in terms of local coordinates $q=(v, \delta, \theta, \omega, x, y)^{T}$ as first "flipping" $(\delta, \theta, \omega, y)$, integrating the left step map and then flipping back, namely

$$
f_{R}(q)=M f_{L}(M q)
$$

where $M=\operatorname{diag}\{1,-1,-1,-1,1,-1\}$. Note that $M M=I$. We chose to flip about the $x$-axis for notational simplicity but in principle any left-handed frame would work. This mapping leaves the right step map left-invariant under SE(2).

For finding symmetric steady-state gaits, it will be convenient to define a special step-to-step return map that amounts to an "integrate and flip" [see Fig. 9(b)]. For a complete stride that includes a left step and then a right step, the stride-to-stride mapping is given by $f_{L-R}=f_{R} \circ f_{L}$, namely

$$
f_{L-R}: q \mapsto M f_{L}\left(M f_{L}(q)\right)=(f \circ f)(q)
$$

where $f(q):=M f_{L}(q)$.

This approach eliminates the need to distinguish between left and right steps for control purposes. Note, however, that $f$ is not left-invariant, even though both $f_{L}$ and $f_{R}$ are left-invariant. The resulting state evolution is given simply by

$$
q_{k+1}=f\left(q_{k}\right)
$$

keeping in mind that, for odd steps, the value for $q_{k}$ in this step-to-step formulation has already been "flipped."

\section{Reduced Return Map}

To simplify controller analysis and design, we use translational symmetry and energy conservation, as first reported in [31]. Recall that the left- and right-step mappings, $f_{L}$ and $f_{R}$, are invariant to $\operatorname{SE}(2)$, but the step-to-step return map, $f=M f_{L}$, is not. However, that mapping is invariant to pure $x$ motions (had we chosen a different left-handed frame, translational invariance would have been in the direction of the axis of symmetry of the reflection to that frame). This was by design: our goal for control is wall following, and for simplicity, we have chosen to follow the $x$-axis. Thus, $x$ is removed by setting $x=0$ at the beginning of each step. To remove $v$, note that the Hamiltonian equation

$$
H=\frac{1}{2} m v^{2}+\frac{1}{2} I \omega^{2}+\frac{1}{2} k\left(\eta-l_{0}\right)^{2}=H_{0}
$$

is constant because the system energy is globally conserved. So, at each step

$$
v=\left[\frac{2}{m}\left(H_{0}-\frac{1}{2} I \omega^{2}-\frac{1}{2} k\left(\eta-l_{0}\right)^{2}\right)\right]^{1 / 2} .
$$

Thus, we have the transformation

$$
T_{H}:(\delta, \theta, \omega, y) \mapsto(v, \delta, \theta, \omega, 0, y)
$$

that assigns $x=0$ and computes $v$ from (18). Note that $T_{H}$ is invertible and $T_{H}^{-1}$ is the transformation that simply removes the $v$-and $x$-coordinates. Then, we define the reduced variables and mapping

$$
q^{r}=(\delta, \theta, \omega, y), \quad f^{r}\left(q_{k}^{r}, u_{k}\right)=T_{H}^{-1}\left(f\left(T_{H}\left(q_{k}^{r}\right), u_{k}\right)\right) .
$$



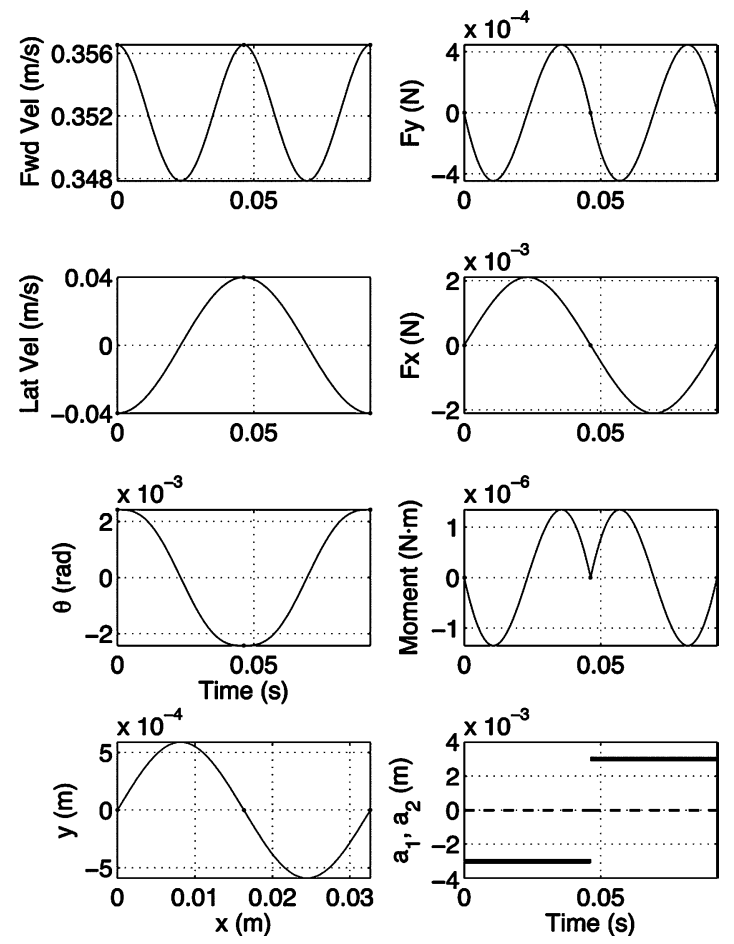

Fig. 11. Full stride of LLS at its equilibrium point with the following parameters: $m=0.77 \times 10^{-3} \mathrm{~kg}, J=1.0 \times 10^{-7} \mathrm{~kg} \cdot \mathrm{m}^{2}, l_{0}=0.0165 \mathrm{~m}$, $k=0.766 \mathrm{~N} / \mathrm{m}, \beta_{0}=1.051 \mathrm{rad}, a_{1}=0 \mathrm{~m}, a_{2}=-0.003 \mathrm{~m}, v(0)=$ $0.359 \mathrm{~m} / \mathrm{s}$. In the last subplot, dashed and solid lines are $a_{1}$ and $a_{2}$, respectively.

\section{Antenna-Based LLS (ALLS) Under PD Control}

In the Appendix, we find the LLS parameters for $P$. americana and show how we simulate the equations of motion for the LLS model; a simulation of LLS using those parameters is shown in Fig. 11. Based on the same antenna model (1) used previously, we (numerically) "embed" the PD-controlled APU template in the LLS model providing a candidate mechanism for leggedlocomotion heading control via antennal feedback.

As a preliminary control task, we chose to have the antennabased LLS (ALLS) follow on top of a line or a virtual "wall" that is coincident with the $x$-axis. The result was an equilibrium point $\bar{q}^{r}=(\bar{\delta}, \bar{\theta}, \bar{\omega}, \bar{y})^{T}$ such that $\bar{q}^{r}=f^{r}\left(\bar{q}^{r}, 0\right)$. To address controllability, we numerically linearized the return map around a nominal equilibrium trajectory, to obtain

$$
e_{k+1}=A e_{k}+B u_{k}, \quad z_{k}=C e_{k}
$$

where $A=\left(\partial f^{r} / \partial q^{r}\right)\left(\bar{q}^{r}, 0\right), \quad B=\left(\partial f^{r} / \partial u\right)\left(\bar{q}^{r}, 0\right), \quad C=$ $\left(\partial h / \partial q^{r}\right)\left(\bar{q}^{r}, 0\right), e_{k}=q_{k}^{r}-\bar{q}^{r}, z_{k}=\left[d_{k}-\bar{d}, \dot{d}_{k}-\dot{\bar{d}}\right]^{T}, u_{k}:=$ $a_{1, k}$, and $h=[d, \dot{d}]^{T}$. We used $a_{1}$ rather than $a_{2}$ as our control input because, in Fig. 10, the gradient of the eigenvalues is greater in the direction of $x$-axis than in the direction of $y$-axis of $\{\mathrm{B}\}$. That is, a small displacement in $a_{1}$ gives us a greater control than that of $a_{2}$; this is consistent with Full and Koditschek's hypothesis, "maneuvers require minor neuromechanical alterations" [4]. In addition, updating the control input once per step (rather than continuously) resonates with the notion that inherent mechanical stability puts less demands on neural feedback [4]. In (21), the condition number for the controllability and observability matrices are 7445 and 390, respectively, so the system is controllable and observable.

Here, we make several approximations to the ALLS model to simplify control and connect the ALLS to a simpler model in our research program (Fig. 1). The third row of the linearized discrete dynamics (21) for parameters for P. americana can be written as

$$
\omega_{k+1}-\omega_{k}=-(1.96) \omega_{k}-(1.08)\left(\delta_{k}-\bar{\delta}\right)+(611.86) u_{k} .
$$

Since our simulations suggest that $\delta_{k}-\bar{\delta}$ remains at least an order of magnitude smaller than the other terms during transients, we neglect $\delta_{k}-\bar{\delta}$ and consider $\dot{\omega} \approx\left(\omega_{k+1}-\omega_{k}\right) f_{s}$, where $f_{s}=10.8 \mathrm{~Hz}$ (see the Appendix) is the stride frequency. Thus, we approximate (22) with a continuous-time system,

$$
\dot{\omega} \approx-\alpha \omega+u^{\prime}
$$

where $\alpha \approx 21.2$ and $u^{\prime} \approx(6608) u_{k}$. This equation mirrors the unicycle model (2), and despite the fairly crude approximations, the coefficient $\alpha \approx 21.2$ in the LLS approximation (23) is within the confidence intervals of the fitted parameters for $\alpha$ in cockroaches (Table I). Also note that the $u$ in (2) is a moment (scaled by inertia), whereas in the ALLS model, the control input is the COP position. Hence, the coefficient multiplying the control $u_{k}$ in (23) is absorbed into $u^{\prime}$ for comparison purposes.

The similarity between the APU (2) and the approximate LLS (23) reveals a possible embedding of the PD-controlled APU into the ALLS. In fact, by setting $u_{k}^{\prime}=-K_{P} d_{k}-K_{D} \dot{d}_{k}$ with the same control parameters fitted to the cockroach yields a closed-loop system of $e_{k+1}=(A+B K C) e_{k}$ with all of its eigenvalues $(-0.64 \pm 0.16 j$ and $-0.13 \pm 0.49 j)$ inside the unit circle, i.e., the closed-loop system is stable around the equilibrium trajectory.

\section{E. Simulation Result}

A simulation for this controller using the parameters for P. americana is shown in Fig. 12(e). In this control law, the COP lies nominally along the body $y$-axis, namely, $a_{1}=0$ and $a_{2}=-3 \mathrm{~mm}$ (for the left step); the feedback controller varies the COP in the $a_{1}$-direction.

The most parsimonious controller sufficient to stabilize highspeed wall following in the APU model is a continuous PD controller mapping antenna measurements to a continuous moment about the COM. As shown, this control law applies with essentially no modification to the control of a legged running model, ALLS, by mapping sensor values to the COP position during each step. This result further supports the hypothesis that such a simple PD controller may underly task-level locomotion control of the American cockroaches.

\section{DISCUSSION}

This paper takes a multifaceted view (Fig. 12) of an extraordinary sensory-feedback-driven locomotor behavior [2]: highspeed antenna-based wall following in the American cockroach. Cowan et al. [1] model this behavior [Fig. 12(a)] as a simple PD controller acting on the APU template [Fig. 12(b)]. Here, 

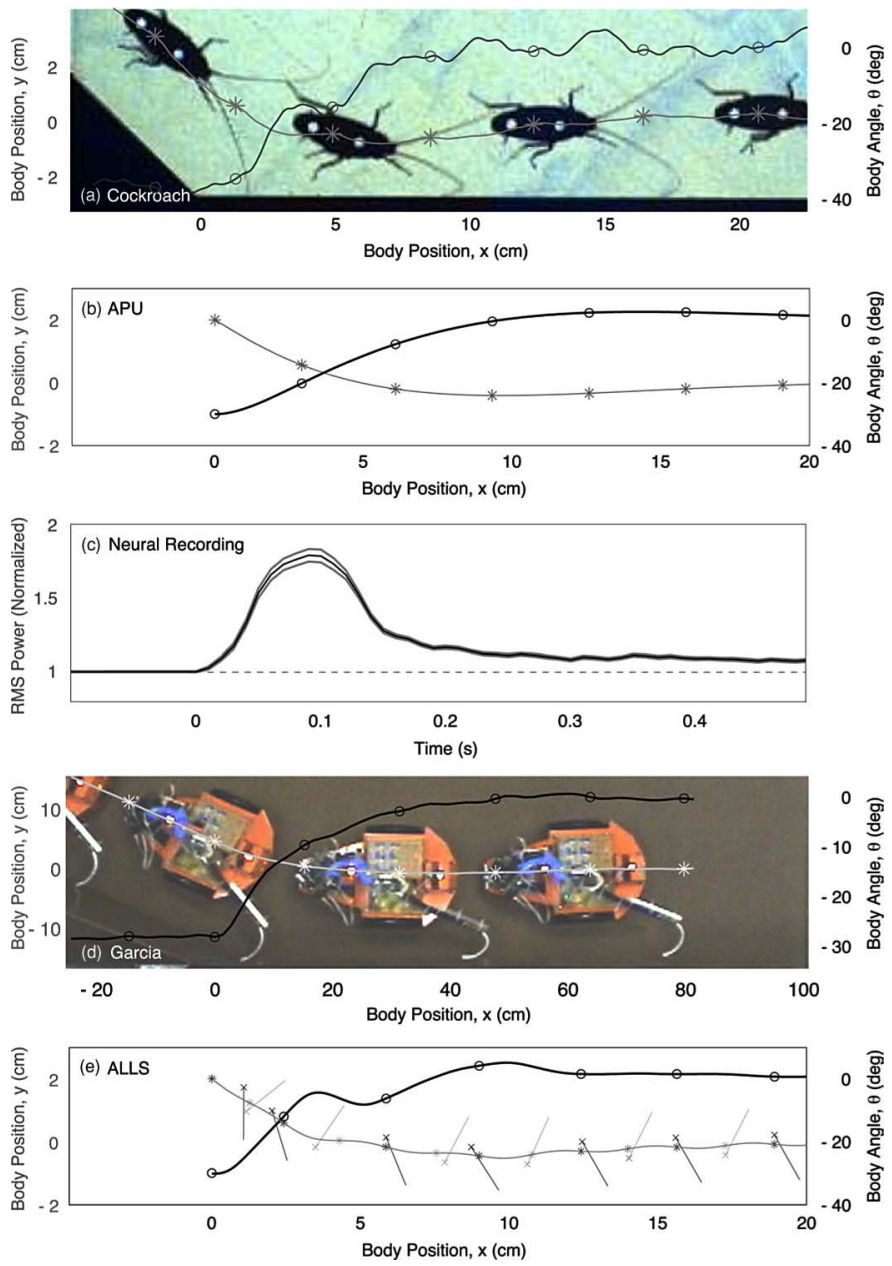

Fig. 12. Subplots (a), (b), (d), and (e) show the task-space trajectories of several models and physical systems from our research program (Fig. 1); *'s and o's indicate COM position and body angle, respectively, at the start of every stride (or dimensionally equivalent stride); (b), (d), and (e) have dimensionally equivalent parameters and the same PD-control law as discussed in the text. (a) Typical trial of $P$. americana (the cockroach is shown every other stride) [1]; the cockroach is following the wall at $\sim 45 \mathrm{~cm} / \mathrm{s}$. (b) APU model simulation using the parameters given in Table I (first row). (c) Neural recording near the base of flagellum. (d) Garcia robot experiment, where the robot is shown every other "stride"; the robot uses parameters given in Table I (second row). (e) ALLS model simulation, shown at the start of every step with *'s indicating COM, $\times$ 's indicating $\mathrm{COP}$, and straight lines emanating from COP indicating the effective leg.

our neural recordings of the antenna [Fig. 12(c)] support the hypothesis that antennal mechanoreceptors can serve as effective inputs to the proposed PD controller because correlates of distance and rate of approach to a wall appear directly in the antennal nerve and the time course of the neural response closely matches the kinematics of turning. Next, we confirm that the PD controller (including the gains) fitted to cockroach behavior is sufficient in a real-world setting: our robot tuned with dimensionally scaled parameters and controller gains stably follows walls using our bio-inspired artificial antenna [Fig. 12(d)], and exhibits transient behavior comparable to both the simulated APU and the cockroach itself. To test the feasibility of the same PD controller in a legged system, we use a modified version of the LLS model [29], [30]—namely, ALLS—in which the controller has authority over the position of the COP at the start of each step as a function of antennal feedback. Importantly, we fit the parameters for the "open-loop" LLS model (leg stiffness, leg touchdown angle, etc.) during steady-state running, independent of the fitted closed-loop APU model from Section II and [1]. Nevertheless, we find that the torsional dynamics of the LLS model can be numerically "reduced" to those of the APU model, with very close agreement in parameters. Using the same PD gains as the APU model, the ALLS model exhibits stability and a similar transient response [Fig. 12(e)] as the cockroach.

\section{A. Multilevel Modeling}

To elucidate the behavior of antenna-based wall following of the American cockroaches, we formulate our research program using templates and anchors [4] (Fig. 1), enabling us to address specific questions at each level in the hierarchy, as well as make quantitative connections between levels. For example, the simplest template, the APU model, neglects within-stride dynamics but, nevertheless, reveals a candidate task-level control law. We then anchor the controlled APU in the Garcia and ALLS models. At the same time, the successive elaboration of features in more complex models (e.g., forward-speed control in the Garcia and within-stride dynamics of the LLS) allows us to address increasingly refined questions about the underlying biological system.

\section{B. Implications From Neural Recordings}

Our neurophysiological recordings of cockroach antennae indicate that the primary biological sensors the cockroach uses to maintain wall following could provide sufficient inputs for an effective PD controller. While P (tonic) and D (phasic) information is known in other biological mechanoreceptors, the population of antennal mechanoreceptors here also appear to filter the mechanical stimulus so that there is close agreement between the time course of the neural activity and the kinematic response of the cockroach, the time evolution of the mathematical models, and the behavior of the robot. For example, despite the deflection of the antenna lasting for only $\sim 40 \mathrm{~ms}$, the phasic response of the receptors peaks at approximately $90 \mathrm{~ms}$, surprisingly close to a typical stride period (Fig. 13). The filtering of the sensory signal in the primary afferents was not expected and differs from many arthropod receptors that are direct velocity or position sensors. Determining whether this property matches individual receptor responses or only emerges from the population of receptors requires extensive individual mechanoreceptor recordings and is beyond the scope of this paper. However, regardless of the mechanism, this slower time response of the neural signal is not likely due to constraints on the encoding process, as many biological mechanoreceptors can transduce very-high-frequency signals [15], [32]. Rather, we suggest this temporal filtering may have a constructive effect, preconditioning the signal to act as an effective PD input tuned to the mechanics of the legged organism. This is consistent with the efficacy of this control model in the ALLS model with cockroach-like dynamics.

The neurophysiology here constitutes only an initial investigation into the neural basis of wall-following maneuvers. The 
phasic and more sustained response within the antennal nerve and the general similarity to the time course of the kinematics of wall following are certainly consistent with the PD-control model generated from the ethological experiments. However, we cannot rule out more complex control models incorporating higher order, nonlinear, or integral terms. Additional neurophysiology and information theoretic analyses could reveal the actual encoding strategies employed by individual mechanosensory neurons. Furthermore, improvements in recording techniques where the activity in individual afferents can be resolved will be useful in determining whether individual afferents encode both $\mathrm{P}$ and $\mathrm{D}$ information, or if this information is segregated between different classes of afferents. These potential organizational features may have important implications for the transformation of the sensory signals into motor commands in downstream circuits.

\section{Biologically Inspired Tactile Sensors}

Toward our goal of a robotic model of wall following, our antenna design captures several key features of cockroach antennae. However, due to the constraints of our prototyping process, the antenna stiffness was not well matched to its biological counterpart: unlike that of the cockroach, this stiffness produced a nonnegligible force between the robot and the wall causing an offset in the robot's distance to the wall. To remedy this issue, we need to better characterize the cockroach antenna mechanics, similar to a prior study of crayfish antennae [33]. Then, we need to design our antenna to match important parameters such as the stiffness profile. We believe that shape deposition manufacturing, also used for manufacturing robot legs [34], [35], offers a viable solution.

In addition to enhancing our inquiry into a biological system, bio-inspired antennae offer substantial benefits to robotics. Ours consists of a ten-dollar $50 \mathrm{k} \Omega$ flex sensor cut into four pieces enclosed in urethane, rendering it inexpensive, low power, and mechanically robust. In addition, our antenna is insensitive to low or extremely bright ambient light (unlike vision and IR), does not emit energy (unlike sonar and IR), and does not require a specific wall type (unlike sonar, IR, and vision, which may fail for common urban surfaces such as fences, highly specular walls, or glass).

Other researchers have built tactile sensors inspired by arthropod antennae and mammalian whiskers. Our research builds directly on [36] that uses a single unmodified flex sensor to control a hexapod on a treadmill. Our design is similar to that used by Barnes et al. [37], who embed three bend sensors in a passive, large-deflection antenna inspired by lobsters that distinguishes between contact with solid objects versus water currents [38]. Whereas our design focuses on control in the horizontal plane, Lewinger et al. [39] use two stiff cockroach-inspired antennae to traverse sagittal plane obstacles. Our antenna uses no basal information, but whisker-inspired devices [25], [40]-[44], by contrast, use only basal information, since whiskers themselves are insensitive hairs [45]. Future development of tactile sensors for robots may benefit from combining the relative advantages from, but not limited to, both mammalian whiskers and arthropod antennae.

\section{Robotics for Biology}

Physical models can provide an important link between biological experimentation and mathematical modeling. In biology, complexity can obscure generalizing principles, and varying parameters to test a system's responses is often difficult and timeconsuming. In addition, it is difficult to capture an animal's internal states (e.g., neural recordings) while minimizing interference with the animal's natural behavior. Mathematical models can reveal idealized responses, but inevitably neglect the complexity of interaction with the surrounding environment. Experimental robotics allows the embodiment of control hypotheses in the context of difficult-to-model real-world phenomena where, in comparison to biology, it is much easier to vary system parameters and monitor system state variables. These can help in generating, refuting, and supporting biological hypotheses [5].

In this paper, we use our robot to support the hypothesis of the efficacy of the PD controller-which is stable in our mathematical model - in a real-world setting. Two further observations can be made: 1) our result supports Camhi and Johnson's [2] claim that cockroach wall following is mediated by the flagellum (not the base) of the antenna: our robot successfully follows a wall using feedback from the two distal flagellar antenna segments; 2) Fig. 8 reveals that the base flex sensor can potentially provide a sensory cue faster than that from the rest of flex sensors, particularly if we account for the typical conduction velocity (1-4 m/s) for nongiant invertebrate neurons. This observation is consistent with the experiment by Comer et al. [20] where they show the important role that the mechanoreceptors at the base of the antenna play in triggering an escape response.

Other researchers have considered robotics to address biological behaviors [46]. For example, Chapman and Webb [3] implement a neural circuit on a mobile robot to test the sufficiency of their neural model in a real-world setting; equipped with IR sensors acting as its "antennae," the robot exhibits an escape response followed by a wall-following behavior, much like that of a cockroach.

\section{E. ALLS for Biology and Robotics}

In this paper, we show that the ALLS model exhibits stable wall following using exactly the same PD gains as the APU [1]. We propose two hypotheses from our result: 1) the afferents or the CNS suppresses (via a low-pass or notch filter) the sensory input frequency near their stride frequency; this hypothesis is motivated by the fact that the sensory signals seem to show a low-pass filtered response consistent with the time course of the stride-to-stride kinematics (Section III). Alternative hypotheses are that: a) an efferent copy might be used to cancel out the frequencies observed by the antenna [13] or b) the mechanics of the antenna alone can filter the oscillation. To test the effect of the lateral oscillations on the antenna filtering, legged robots such as RHex [47] or even wheeled robots such as Garciaemulating, up to some limit, the within-stride dynamics of the ALLS - could be used. 2) Increasingly anchored models that 
represent cockroach kinematics with increasing biofidelity can be used to tease apart the contributions of individual legs during turning [11]. We hypothesize that the motion of the COP from step to step is governed by our PD controller. To test this, an experimental paradigm may consist of cockroaches following along a wall with perturbations [1], [2] while individual leg forces and kinematics are measured to recover COP motions [48]. Together, these data could be used to approximate the mapping from antennal measurements to COP motions.

In this paper, we numerically reduce the ALLS model to represent the APU's PD controller for the ALLS. A more formal reduction is warranted. For example, Poulakakis and Grizzle [49] provide a formal approach to apply a controller defined for a spring-loaded inverted pendulum (SLIP) model of sagittalplane running to a more anchored model. Similarly, it may be interesting to address how the PD controller for the APU model would be applied to its higher anchors such as ALLS, ALLS with three legs [50], or 3-D SLIP/ALLS model [51]-[53]. This may help in generating hypotheses such as the placement of the COP in 3-D that can generate motions not only in yaw but also in pitch and roll.

With this biological understanding, we will be poised to create bio-inspired control strategies for hexapedal robots [47] by shifting their COP based on sensory stimuli. The shifting of the COP can be achieved by touching down three legs that collectively generate a force vector pointed at the desired COP position, similar to that of cockroaches [11], [48]. The sagittalplane motions that result from 3-D models or legged robots may lead to important design requirements for artificial sensors; for example, the antenna may be need to be stiffer along the sagittal plane than along the lateral plane [13], [54], [55].

\section{CONCLUSION}

In this paper, we show that the synergy between robotics and biology enables mutual discoveries for both fields. Specifically, we take a multidisciplinary approach that incorporates mathematical modeling, robotic experiments, ethology, and neurophysiology to provide a glimpse into the neuromechanical control of one of the fastest terrestrial insects, the American cockroach [56]. Along the way, we develop a novel bio-inspired tactile sensor that can be used for high-speed wall following in robotics. Furthermore, our biological modeling reveals a new idea for the control of legged robots under sensory feedback: stride-to-stride COP placement may provide a simple mechanism for task-level control based on sensory feedback. The implementation of the legged robot control based on this idea may, in turn, help biology by providing a new vehicle to test and help generate specific interleg coordination strategies for modulating the COP, creating further opportunities for discovery in both robotics and biology.

\section{APPENDIX}

\section{A. ALLS Simulation Methods}

We simulate the LLS model using Matlab (The MathWorks, Inc., Natick, MA) using the convention in Section V-B: for
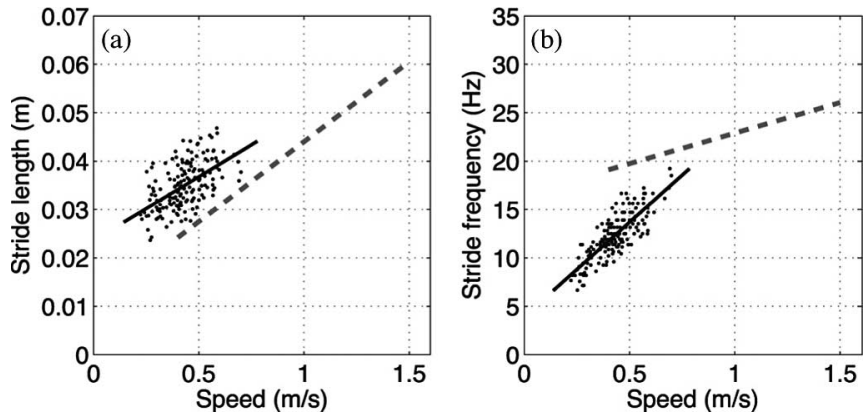

Fig. 13. (a) Stride length versus speed and (b) stride frequency versus speed of the first four strides (prior to angled-wall contact) of 43 trials from the data that Cowan et al. [1] have collected. The solid line is the linear fit of the data. The dashed line is the linear fit of the data collected by Full and Tu [57]. Unlike Full and Tu, Cowan et al. use cockroaches that are blinded, in an escaperesponse mode, and following a wall; this difference in the behavioral context may account for the observed differences in running speeds between the two groups [19]. We assume that the data are adequate for our preliminary fitting of the LLS model, and we reduce the bias incurred from wall following by matching the waveform characteristics (e.g., stride length and stride frequency) rather than the waveform itself.

every right-leg step, convert it to a left-leg step, simulate the within-step dynamics, and then, convert it back to a right-leg step. This enables us to specify the COP position using (11) and integrate the equations of motion derived from (13) without the explicit representations of a left or right step in the equations. We use Matlab's ode45 with time-varying step size to integrate the equations of motion. The integration for a step terminates as soon as the compressed leg returned back to its relaxed length $l_{0}$.

We find the equilibrium point $\bar{q}=(v, \delta, \theta, \omega, y)^{T}$ using the Levenberg-Marquardt method in Matlab's fsolve function. While fixing the state $v$ to a desired value, the function minimizes the error difference of a step $f(q)-q$. We find the step-to-step return map Jacobian $A$ about the equilibrium point using a central difference approximation. The $i$ th columns are given by $[A]_{i}=\left(f^{r}\left(q+e_{i} \epsilon\right)-f^{r}\left(q-e_{i} \epsilon\right)\right) / 2 \epsilon$, where $\epsilon=1 \times 10^{-6}$ and $e_{i}$ is the $i$ th column of $4 \times 4$ identity matrix. Similarly, we find the stride-to-stride return map Jacobian to determine the stride-to-stride eigenvalues for Fig. 10.

We use the following parameters and measurements of $P$. americana: $m=0.77 \times 10^{-3} \mathrm{~kg}, J=1.0 \times 10^{-7} \mathrm{~kg} \cdot \mathrm{m}^{2}, \bar{v}$ $($ average velocity $)=0.352 \mathrm{~m} / \mathrm{s}, L_{s}($ stride length $)=0.033 \mathrm{~m}$, $f_{s}($ stride frequency $)=10.8 \mathrm{~Hz}, v_{\text {lateral, } \max }=0.04 \mathrm{~m} / \mathrm{s}$, and $\theta_{\max }=0.03 \mathrm{rad}$. We use the rectangular prism approximation [48] to find the moment of inertia in the yaw direction. The stride length and the stride frequency has been found from Fig. 13.

The values for leg rest length $l_{0}$, leg spring constant $k$, initial leg touchdown angle $\beta_{0}$, and fixed COP position $a_{2}$ are chosen to satisfy constraints on the stride length, stride frequency, and maximum lateral velocity. Because the system is underdetermined, we make sure that these parameters are within an order of magnitude from the dimensionally scaled values of Blaberus discoidalis used in [8]. A simulation of LLS model using this set of parameter values is shown in Fig. 11. The magnitude of the body oscillation is about an order of magnitude smaller than that of the actual cockroach because of LLS's simplification of the tripod legs into a single virtual leg; a single leg cannot generate 
enough torque to match that of the original three legs while having the parameter values that are physically realizable [8], [31].

\section{ACKNOWLEDGMENT}

The authors thank B. Kutscher for help with software and hardware integration and J. Schmitt for providing insight into the LLS model. They also thank E. Roth, V. Kallem, E. Fortune, S. Carver, N. Keller, K. Canfield, and four anonymous biology and engineering reviewers for providing critical reviews of the manuscript. They would further like to thank Dr. A. Spence, E. Hebets, J. Miller, and A. Vaughan.

\section{REFERENCES}

[1] N. J. Cowan, J. Lee, and R. J. Full, "Task-level control of rapid wall following in the American cockroach," J. Exp. Biol., vol. 209, no. 9, pp. 1617-1629, 2006.

[2] J. M. Camhi and E. N. Johnson, "High-frequency steering maneuvers mediated by tactile cues: Antenna wall-following in the cockroach," $J$. Exp. Biol., vol. 202, pp. 631-643, 1999.

[3] T. P. Chapman and B. Webb, "A model of antennal wall-following and escape in the cockroach," J. Comp. Physiol. A, vol. 192, no. 9, pp. 949969, Sep. 2006.

[4] R. J. Full and D. E. Koditschek, "Templates and anchors: Neuromechanical hypotheses of legged locomotion on land," J. Exp. Biol., vol. 202, no. 23, pp. 3325-3332, 1999.

[5] P. Holmes, R. J. Full, D. Koditschek, and J. Guckenheimer, "The dynamics of legged locomotion: Models, analyses, and challenges," SIAM Rev., vol. 48, no. 2, pp. 207-304, 2006.

[6] T. M. Kubow and R. J. Full, "The role of the mechanical system in control: A hypothesis of self-stabilization in hexapedal runners," Philos. Trans. R. Soc. B, vol. 354, no. 1385, pp. 849-861, 1999.

[7] D. L. Jindrich and R. J. Full, "Dynamic stabilization of rapid hexapedal locomotion," J. Exp. Biol., vol. 205, no. 18, pp. 2803-2823, 2002.

[8] J. Schmitt, M. Garcia, R. C. Razo, P. Holmes, and R. J. Full, "Dynamics and stability of legged locomotion in the horizontal plane: A test case using insects," Biol. Cybern., vol. 86, pp. 343-353, 2002.

[9] J. E. Seipel and P. Holmes, "Running in three dimensions: Analysis of a point-mass sprung-leg model," Int. J. Robot. Res., vol. 24, pp. 657-674, 2005.

[10] R. J. Full, T. M. Kubow, J. Schmitt, P. Holmes, and D. E. Koditschek, "Quantifying dynamic stability and maneuverability in legged locomotion,” Integr. Comp. Biol., vol. 42, no. 1, pp. 149-157, 2002.

[11] D. L. Jindrich and R. J. Full, "Many-legged maneuverability: Dynamics of turning in hexapods," J. Exp. Biol., vol. 202, no. 12, pp. 1603-1623, 1999.

[12] G. Seelinger and T. R. Tobin, "Sense organs," in The American Cockroach. London, U.K.: Chapman and Hall, 1982, pp. 217-245.

[13] E. Staudacher, M. Gebhardt, and V. Dürr, "Antennal movements and mechanoreception: Neurobiology of active tactile sensors," Adv. Insect. Physiol., vol. 32, pp. 49-205, 2005.

[14] J. Okada and Y. Toh, "The role of antennal hair plates in object-guided tactile orientation of the cockroach (Periplaneta americana)," J. Comp. Physiol. A, vol. 186, no. 9, pp. 849-857, 2000.

[15] J. Okada and Y. Toh, "Peripheral representation of antennal orientation by the scapal hair plate of the cockroach Periplaneta americana," J. Exp. Biol., vol. 204, pp. 4301-4309, 2001.

[16] J. Okada, Y. Kanamaru, and Y. Toh, "Mechanosensory control of antennal movement by the scapal hair plate in the American cockroach," Zool. Sci., vol. 19, no. 11, pp. 1201-1210, Nov. 2002.

[17] C. M. Comer and J. P. Dowd, "Multisensory processing for movement: Antennal and cercal mediation of escape turning in the cockroach," in Biological Neural Networks in Invertebrate Neuroethology and Robotics, R. D. Beer, R. E. Ritzmann, and T. McKenna, Eds., New York: Academic, 1993, pp. 89-112.

[18] C. Comer, E. Mara, K. A. Murphy, M. Getman, and M. C. Mungy, "Multisensory control of escape in the cockroach Periplaneta americana. II. Patterns of touch-evoked behavior," J. Comp. Physiol. A, vol. 174, pp. 1326, 1994.

[19] S. Ye, V. Leung, A. Khan, Y. Baba, and C. M. Comer, "The antennal system and cockroach evasive behavior. I. Roles for visual and mechanosen- sory cues in the response," J. Comp. Physiol. A, vol. 189, pp. 89-96, 2003.

[20] C. M. Comer, L. Parks, M. B. Halvorsen, and A. Breese-Terteling, "The antennal system and cockroach evasive behavior. II. Stimulus identification and localization are separable antennal functions," J. Comp. Physiol. A, vol. 189, pp. 97-103, 2003.

[21] Y. Toh, "Fine structure of antennal sense organs of the male cockroach, Periplaneta americana," J. Ultrastruct. Res., vol. 60, pp. 373-394, 1977.

[22] K. M. Chapman and J. H. Pankhurst, "Conduction velocities and their temperature coefficients in sensory nerve fibres of cockroach legs," $J$. Exp. Biol., vol. 46, pp. 63-84, 1967.

[23] R. Foelix, D. Troyer, and P. Igelmund, "Peripheral synapses and giant neurons in whip spiders," Microsc. Res. Tech., vol. 58, no. 4, pp. 272 282, Aug. 2002.

[24] R. Schafer and T. V. Sanchez, "Antennal sensory system of the cockroach Periplaneta americana: Postembryonic development and morphology of the sense organs," J. Comp. Neurol., vol. 149, pp. 335-354, 1973.

[25] A. E. Schultz, J. H. Solomon, M. A. Peshkin, and M. J. Hartmann, "Multifunctional whisker arrays for distance detection, terrain mapping, and object feature extraction," in Proc. IEEE Int. Conf. Robot. Autom., Apr. 18-22, 2005, pp. 2588-2593.

[26] G. R. Scholz and C. D. Rahn, "Profile sensing with an actuated whisker," IEEE Trans. Robot. Autom., vol. 20, no. 1, pp. 124-127, Feb. 2004.

[27] A. Lamperski, O. Loh, B. Kutscher, and N. J. Cowan, "Dynamical wallfollowing for a wheeled robot using a passive tactile sensor," in Proc. Int. Conf. Robot. Autom., 2005, pp. 3838-3843.

[28] D. M. Dudek and R. J. Full, "Passive mechanical properties of legs from running insects," J. Exp. Biol., vol. 209, no. 8, pp. 1502-1515, 2006.

[29] J. Schmitt and P. Holmes, "Mechanical models for insect locomotion: Dynamics and stability in the horizontal plane-I. Theory," Biol. Cybern., vol. 83, pp. 501-515, 2000.

[30] J. Schmitt and P. Holmes, "Mechanical models for insect locomotion: Dynamics and stability in the horizontal plane-II. Application," Biol. Cybern., vol. 83, pp. 517-527, 2000.

[31] J. Lee, A. Lamperski, J. Schmitt, and N. J. Cowan, "Task-level control of the lateral leg spring model of cockroach locomotion," in Fast Motions in Biomechanics and Robotics: Optimization and Feedback Control (Lecture Notes in Control and Information Sciences), M. Diehl and K. Mombaur, Eds. Heidelberg, Germany: Springer-Verlag, 2006, vol. 340, pp. 167188.

[32] F. G. Barth, "Spider mechanoreceptors," Curr. Opin. Neurobiol., vol. 14, no. 4, pp. 415-422, Aug. 2004

[33] R. C. Taylor, "Physical and physiological properties of the crayfish antennal flagellum," J. Neurobiol., vol. 6, no. 5, pp. 501-519, Sep. 1975.

[34] J. G. Cham, S. A. Bailey, J. E. Clark, R. J. Full, and M. R. Cutkosky, "Fast and robust: Hexapedal robots via shape deposition manufacturing," Int. J. Robot. Res., vol. 21, no. 10, pp. 869-882, 2002.

[35] A. T. Asbeck, S. Kim, M. R. Cutkosky, W. R. Provancher, and M. Lanzetta, "Scaling hard vertical surfaces with compliant microspine arrays," Int. J. Robot. Res., vol. 25, no. 12, pp. 1165-1179, 2006.

[36] N. J. Cowan, E. J. Ma, M. Cutkosky, and R. J. Full, “A biologically inspired passive antenna for steering control of a running robot," in Robotics Research (Springer Tracts in Advanced Robotics). Siena, Italy: SpringerVerlag, 2003, pp. 540-550.

[37] T. Barnes, T. Truong, G. Adams, and N. McGruer, "Large deflection analysis of a biomimetic lobster robot antenna due to contact and flow," Trans. ASME, vol. 68, pp. 948-951, 2001.

[38] J. Ayers, "A reactive ambulatory robot architecture for operation in current and surge," in Proc. Auton. Veh. Mines Countermeasures Symp., 1995, pp. $15-31$.

[39] W. A. Lewinger, C. M. Harley, R. E. Ritzmann, M. S. Branicky, and R. D. Quinn, "Insect-like antennal sensing for climbing and tunneling behavior in a biologically-inspired mobile robot," in Proc. IEEE Int. Conf. Robot. Autom., Apr. 2005, pp. 4176-4181.

[40] R. A. Russell, "Object recognition using articulated whisker probes," in Proc. Int. Symp. Intell. Robot. Tokyo, Japan, 1985, pp. 605-611.

[41] D. Jung and A. Zelinsky, "Whisker-based mobile robot navigation," in Proc. IEEE/RSJ Int. Conf. Intell. Robots Syst., Nov. 1996, vol. 2, pp. 497504.

[42] M. Kaneko, N. Kanayama, and T. Tsuji, "Active antenna for contact sensing," IEEE Trans. Robot. Autom., vol. 14, no. 2, pp. 278-291, Feb. 1998.

[43] N. Ueno, M. Svinin, and M. Kaneko, "Dynamic contact sensing by flexible beam," IEEE/ASME Trans. Mech., vol. 3, no. 4, pp. 254-263, Dec. 1998 . 
[44] M. Lungarella, V. V. Hafner, R. Pfeifer, and H. Yokoi, "An artificial whisker sensor for robotics," in Proc. 15th IEEE/RSJ Int. Conf. Intell, Robots Syst., Lausanne, Switzerland, 2002, pp. 2931-2936.

[45] M. J. Hartmann, N. J. Johnson, R. B. Towal, and C. Assad, "Mechanical characteristics of rat vibrissae: Resonant frequencies and damping in isolated whiskers and in the awake behaving animal," J. Neurosci., vol. 23, no. 16 , pp. 6510-6519, 2003.

[46] B. Webb, "Can robots make good models of biological behaviour?," Behav. Brain Sci., vol. 24, no. 6, pp. 1033-1050, Dec. 2001 (discussion: 1050-1094).

[47] U. Saranli, M. Buehler, and D. E. Koditschek, "RHex: A simple and highly mobile hexapod robot," Int. J. Robot. Res., vol. 20, no. 7, pp. 616-631, 2001.

[48] L. H. Ting, R. Blickhan, and R. J. Full, "Dynamic and static stability in hexapedal runners," J. Exp. Biol., vol. 197, pp. 251-269, 1994.

[49] I. Poulakakis and J. W. Grizzle, "Formal embedding of the spring loaded inverted pendulum in an asymmetric hopper," presented at the Eur. Control Conf., Kos, Greece, Jul. 2007.

[50] J. Seipel, P. Holmes, and R. Full, "Dynamics and stability of insect locomotion: A hexapedal model for horizontal plane motions," Biol. Cybern., vol. 91, no. 2, pp. 76-90, 2004.

[51] J. Seipel and P. Holmes, "Three-dimensional translational dynamics and stability of multi-legged runners," Int. J. Robot. Res., vol. 25, no. 9, pp. 889-902, Sep. 2006.

[52] U. Saranli, "SimSect hybrid dynamical simulation environment," Univ. Michigan, Ann Arbor, Tech. Rep. CSE-TR-436-00, 2000.

[53] U. Saranli and D. E. Koditschek, "Template based control of hexapedal running," in Proc. IEEE Int. Conf. Robot. Autom., Taipei, Taiwan, Sep. 2003, vol. 1, pp. 1374-1379.

[54] D. C. Sandeman, "Physical properties, sensory receptors and tactile reflexes of the antenna of the australian freshwater crayfish cherax destructor," J. Exp. Biol., vol. 141, no. 1, pp. 197-217, 1989.

[55] C. Loudon, "Flexural stiffness of insect antennae," Amer. Entomol., vol. 51, pp. 48-49, 2005

[56] T. M. Merritt. (1999). Fastest runner, in Book of Insect Records. Univ. of Florida, Gainesville [Online]. Available: http://ufbir.ifas.ufl.edu/

[57] R. J. Full and M. S. Tu, "Mechanics of a rapid running insect: Two-, four-, and six-legged locomotion," J. Exp. Biol., vol. 156, pp. 215-231, 1991.

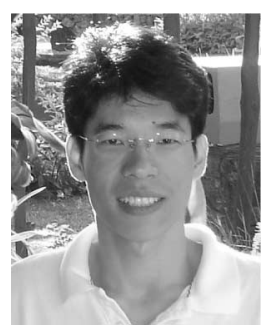

Jusuk Lee received the B.S. degree in electrical engineering and computer sciences from the University of California (UC) at Berkeley, Berkeley, in 2002, and the M.S. degree in mechanical engineering in 2005 from the Johns Hopkins University, Baltimore, MD, where he is currently working toward the Ph.D. degree in mechanical engineering.

He was with the UC Berkeley, where he was engaged in microfactories at the Robotics and Intelligent Machines Laboratory, unmanned aerial vehicles at the Vehicle Dynamics Laboratory, and animal locomotion at the Poly-PEDAL Laboratory. He is currently with the Locomotion in Mechanical and Biological Systems (LIMBS) Laboratory, Johns Hopkins University. His current research interests include animal locomotion and bio-inspired robotics.

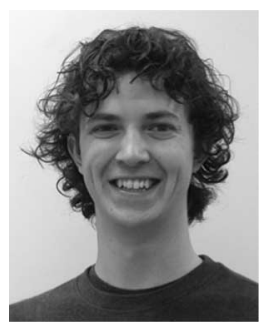

Simon N. Sponberg received the Bachelor's degree in physics and biology at Lewis and Clark College, Portland, OR, in 2002. He is currently working toward the Ph.D. degree at the Poly-PEDAL Laboratory and the Center for Integrative Biomechanics in Education and Research (CIBER), Department of Integrative Biology, University of California at Berkeley, Berkeley.

His current research interests include the neuromechanical basis for stability and maneuverability in biological and bio-inspired systems. He was involved in testing models of passive dynamic stability in rapidly running cockroaches and how sensory feedback information can be integrated with these steady-state dynamics to produce enhanced stability and navigation.

Mr. Sponberg is supported by a Fannie and John Hertz Foundation Graduate Fellowship.

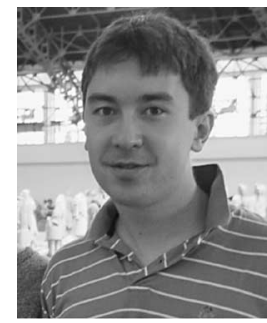

Owen Y. Loh received the B.S. degree in mechanical engineering from the Johns Hopkins University, Baltimore, MD, in 2005. He is currently working toward the Ph.D. degree in mechanical engineering at the Northwestern University, Evanston, IL.

He was at the Johns Hopkins University, where he was engaged in developing biologically inspired tactile sensors at the LIMBS Laboratory. His current research interests include feedback-controlled carbon nanotube-based nanoelectromechanical systems and application of novel nanopatterning techniques to study nanomaterial-mediated cancer drug delivery and its effect on healthy and cancerous cells.

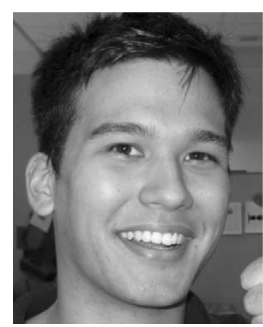

Andrew G. Lamperski received the B.S. degree in biomedical engineering and mathematics from the Johns Hopkins University, Baltimore, MD, in 2004. $\mathrm{He}$ is currently working toward the Ph.D. degree in control and dynamical systems at the California Institute of Technology, Pasadena.

He worked on biologically inspired robots at the LIMBS Laboratory of Johns Hopkins University during the 2003-2004 academic year. His current research interests include hybrid systems and polynomial optimization.

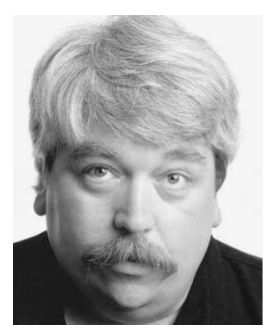

Robert J. Full received the B.A., Master's, and Ph.D. degrees in biological sciences from the State University of New York (SUNY) Buffalo, Buffalo, in 1979, 1982, and 1984, respectively.

From 1984 to 1986, he was a Postdoctoral Researcher at the University of Chicago. In 1986, he joined the faculty of the University of California at Berkeley, Berkeley, as an Assistant Professor of zoology, and became an Associate Professor of integrative biology in 1991, a Full Professor of integrative biology in 1995, and a Chancellor's Professor in 1997. He also directs the Poly-PEDAL Laboratory, which studies the performance, energetics and dynamics of animal locomotion (PEDAL) in manyfooted creatures (Poly). He has been involved in a focused international effort to demonstrate the value of integrative biology and biological inspiration by the formation of interdisciplinary collaborations among biologists, engineers mathematicians, and computer scientists from academia and industry. His fundamental discoveries in animal locomotion have inspired the design of novel neural control circuits, artificial muscles, eight autonomous legged robots, and the first self-cleaning dry adhesive.

Prof. Full received a Goldman Professorship for innovative teaching in 1998. He was recently named a Mentor in the Life Sciences by the National Academy of Sciences.

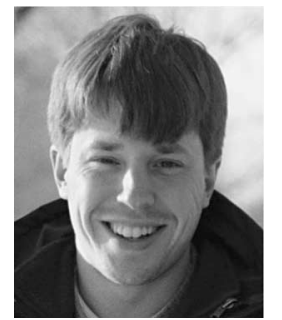

Noah J. Cowan (S'98-M'01) received the B.S. degree in electrical engineering from the Ohio State University, Columbus, in 1995, and the M.S. and $\mathrm{Ph} . \mathrm{D}$. degrees in electrical engineering and computer science from the University of Michigan, Ann Arbor, in 1997 and 2001, respectively.

From 2001 to 2003, he was a Postdoctoral Fellow of both integrative biology and computer science at the University of California, Berkeley. In 2003, he joined the faculty of the Johns Hopkins University, Baltimore, MD, as an Assistant Professor of mechanical engineering, and is currently the Director of the Locomotion in Mechanical and Biological Systems (LIMBS) Laboratory. His current research interests include multisensory control of locomotion in robotics and biology, bio-inspired robotics, and vision-based control with application to medical robotics.

Prof. Cowan received the William H. Huggins Award for excellence in teaching in 2004. He is also the recipient of a Rackham Doctoral Fellowship from the University of Michigan. 